CIRJE-F-824

\title{
An Asymptotic Expansion with Push-Down of Malliavin Weights
}

\author{
Akihiko Takahashi \\ University of Tokyo \\ Toshihiro Yamada \\ Mitsubishi UFJ Trust Investment Technology Institute Co.,Ltd. (MTEC)
}

November 2011; Revised in February 2012

CIRJE Discussion Papers can be downloaded without charge from:

http://www.cirje.e.u-tokyo.ac.jp/research/03research02dp.html

Discussion Papers are a series of manuscripts in their draft form. They are not intended for circulation or distribution except as indicated by the author. For that reason Discussion Papers may not be reproduced or distributed without the written consent of the author. 


\title{
An Asymptotic Expansion with Push-Down of Malliavin Weights *
}

\author{
Akihiko Takahashi ${ }^{\dagger}$ and Toshihiro Yamada ${ }^{\ddagger}$ \\ December 1, 2009; Current Version: December, 2011
}

\begin{abstract}
This paper derives asymptotic expansion formulas for option prices and implied volatilities as well as the density of the underlying asset price in multi-dimensional stochastic volatility models. In particular, the integration-byparts formula in Malliavin calculus and the push-down of Malliavin weights are effectively applied. We provide an expansion formula for generalized Wiener functionals and closed-form approximation formulas in stochastic volatility environment. In addition, we present applications of the general formula to expansions of option prices for the shifted log-normal model with stochastic volatility. Moreover, with some results of Malliavin calculus in jump-type models, we derive an approximation formula for the jump-diffusion model in stochastic volatility environment. Some numerical examples are also shown.
\end{abstract}

Keywords: Malliavin calculus, Asymptotic expansion, Stochastic volatility, Implied volatility, Shifted log-normal model, Jump-diffusion model, Integration-by-parts, Malliavin weight, Push-down, Malliavin calculus for Poisson processes

\section{Introduction}

This paper develops an asymptotic expansion method for generalized Wiener functionals by applying Malliavin weight (divergence given by the integration-by-parts formula) and push-down (the conditional expectation in Malliavin (1997) and Malliavin-Thalmaier (2006)). As applications, we propose a concrete approximation formula of option prices as well as the density of the underlying asset price in multi-dimensional stochastic volatility models, and then derives a new Taylor expansion formula of the implied volatilities. Moreover, we present applications of the general formula to expansions of option prices for the shifted log-normal model in stochastic volatility environment. Also, combining some results of Malliavin calculus in jump-type models by Bavouzet-Messaoud (2006) with our method, we derive an approximation formula for option prices in the jump-diffusion model with stochastic volatility. To the best of our knowledge, it is the first study with push-down of Malliavin weights for deriving analytical approximation formulas for option prices and implied volatilities in those models. A companion paper, Shiraya-Takahashi-Yamada (2009) applies the method to deriving a concrete approximation formula for valuation of barrier options with discrete monitoring under stochastic volatility models.

Various stochastic volatility models has been proposed for calibration to market prices of options with so called volatility skews and smiles. However, closed-form solutions for option prices in the stochastic volatility environment are rarely found, and hence a large number of studies have been made in order to obtain analytical approximations and efficient numerical schemes for option prices and implied volatilities with stochastic volatility models. Takahashi $(1995,1999)$ proposed approximation formulas based on the asymptotic expansion method in Watanabe theory (Watanabe (1983, 1984, 1987), Yoshida (1992 a, b)) for valuation of various derivatives including options in stochastic volatility environment. Fournié et al. (1997) provided an expansion result of the second-order partial differential equation which satisfies the uniform ellipticity condition and showed its application to Monte Carlo simulations. Fouque et al. (2000) derived a closed form approximation formula for the fast mean reverting stochastic volatility model using a singular perturbation method, and then discussed the calibration problem. Hagan et al. (2002) introduced the SABR stochastic volatility model and obtained an approximation implied volatility formula. Labordére (2008) generalized SABR model to $\lambda$-SABR model and derived an approximation of implied volatilities by applying the heat kernel expansion. Moreover, Gatheral et al. (2009) and Ben Arous-Laurence (2009) presented novel results for this direction. Recently, Antonelli-Scarlatti (2009) gave a Taylor series expansions of option prices with respect to a correlation parameter in a stochastic volatility model. Also, we will provide more detail comments on some related works in Section 3.3.

*Forthcoming in SIAM Journal on Financial Mathematics. All the contents expressed in this research are solely those of the authors and do not represent the view of MTEC Co., Ltd. or any other institutions. The authors are not responsible or liable in any manner for any losses and/or damages caused by the use of any contents in this research.

${ }^{\dagger}$ Graduate School of Economics, the University of Tokyo

${ }_{\ddagger}$ Mitsubishi UFJ Trust Investment Technology Institute Co.,Ltd. (MTEC). 
The organization of the paper is as follows: the next section derives an asymptotic expansion formula for generalized Wiener functionals after a brief summary of Malliavin calculus necessary for the remaining of the paper. Section 3 applies the general formula to pricing options in stochastic volatility environment and then obtains implied volatilities' expansions. Section 4 presents numerical examples for expansions including calibrations of implied volatilities. As simple applications of our method, Section 5 provides approximation formulas for option prices (or/and implied volatilities) in the shifted log-normal and jump-diffusion models under stochastic volatilities. Section 6 concludes. Appendix gives explicit calculations of push-down of Malliavin weights (coefficients in the expansions) as well as the proof of Proposition 5.2.

\section{Asymptotic Expansion}

\subsection{Malliavin calculus}

This subsection summarizes basic facts on the Malliavin calculus which are necessary for the following discussion. We use the notations and definitions given below.

Let $(\mathcal{W}, \mu)$ be the Wiener space, i.e.

$$
\mathcal{W}=\mathcal{W}^{d}=C_{0}\left([0, T]: \mathbf{R}^{d}\right)=\left\{w:[0, T] \rightarrow \mathbf{R}^{d} ; \text { continuous, } w(0)=0\right\}
$$

and $\mu$ is the Wiener measure. Next, let $H$ be a Hilbert space such that

$$
H=\left\{h \in \mathcal{W} ; h_{i}(t)(i=1, \cdots, d) \text { is absolute continuous with respect to } t \text { and } \sum_{i=1}^{d} \int_{0}^{T}\left|\frac{d h_{i}(t)}{d t}\right|^{2} d t<\infty\right\}
$$

with an inner product $\left\langle h, h^{\prime}\right\rangle_{H}=\sum_{i=1}^{d} \int_{0}^{T} \frac{d h_{i}(t)}{d t} \frac{d h_{i}^{\prime}(t)}{d t} d t$. Then, $H$ is called the Cameron-Martin space.

Define $L^{\infty-}(\mathcal{W})$ as $L^{\infty-}(\mathcal{W})=\cap_{p<+\infty} L^{p}(\mathcal{W})$ and a distance on $L^{\infty-}(\mathcal{W})$ as

$$
d_{L^{\infty-}(\mathcal{W})}\left(F_{1}, F_{2}\right)=\sum_{j=1}^{\infty} 2^{-j}\left(\min \left\{\left\|F_{1}-F_{2}\right\|_{L^{j}}, 1\right\}\right),
$$

where $\|\cdot\|_{L^{p}}$ denotes the $L^{p}$-norm in $(\mathcal{W}, \mu)$. Given a separable Hilbert space $G$, let $L^{p}(\mathcal{W}: G)$ denote the space of measurable maps from $\mathcal{W}$ to $G$ such that $\|f\|_{G} \in L^{p}(\mathcal{W})$. The same definition is made for $L^{\infty-}(\mathcal{W}: G)$.

Then, consider the space

$$
\begin{aligned}
& \mathbf{D}_{1}^{p}(\mathcal{W}: G) \\
= & \left\{F \in L^{p}(\mathcal{W}: G): \text { there exists } D F \in L^{p}(\mathcal{W}: H \otimes G) \text { such that for } h \in H, \lim _{\epsilon \rightarrow 0} \frac{1}{\epsilon}[F(w+\epsilon h)-F(w)]=(D F(w))(h)\right\} .
\end{aligned}
$$

Here, $D F$ is called the (Malliavin) derivative of $F$. Note also that the tensor product $H \otimes G$ of two separable Hilbert spaces $H$ and $G$ is a Hilbert space formed of all linear operators $A: H \rightarrow G$ of Hilbert-Schmidt type endowed with the Hilbert-Schmidt norm

$$
\|A\|_{H S}=\left(\sum_{i, j=1}^{\infty}\left\langle A e_{i}, e_{j}^{\prime}\right\rangle_{G}^{2}\right)^{1 / 2}
$$

for some ONB(orthonormal basis)'s $\left\{e_{i}\right\}$ in $H$ and ONB's $\left\{e_{j}^{\prime}\right\}$ in $G$, and

$$
\|A\|_{H S}^{2}=\|A\|_{H \otimes G}^{2} .
$$

Then, the random variable $D F$ takes values in $H \otimes G$.

Due to a canonical identification of the Hilbert space $L^{2}(\mathcal{W}: H)$ and $L^{2}([0, T] \times \mathcal{W})$, the Malliavin derivative $D F$ may be considered as a stochastic process $\left\{D_{t} F=\left(D_{t, 1} F, \cdots, D_{t, d} F\right): t \in[0, T]\right\}$ such that

$$
(D F(w))(h)=\langle D F, h\rangle_{H}=\sum_{i=1}^{d} \int_{0}^{T}\left(D_{t, i} F\right)\left(\frac{d h_{i}(t)}{d t}\right) d t .
$$

A norm $\mathbf{D}_{1}^{p}(\mathcal{W}: G)$ is given by $\|F\|_{\mathbf{D}_{1}^{p}(\mathcal{W}: G)}=\|F\|_{L^{p}(\mathcal{W}: G)}+\|D F\|_{L^{p}(\mathcal{W}: H \otimes G)}$. Also, $\mathbf{D}_{1}^{\infty-}(\mathcal{W}: G)$ is defined by $\mathbf{D}_{1}^{\infty-}(\mathcal{W}: G):=\cap_{1 \leq p<+\infty} \mathbf{D}_{1}^{p}(\mathcal{W}: G)$, and a distance on $\mathbf{D}_{1}^{\infty-}(\mathcal{W}: G)$ is given by

$$
d_{\mathbf{D}_{1}^{\infty-}(\mathcal{W}: G)}\left(F_{1}, F_{2}\right)=\sum_{j=1}^{\infty} 2^{-j}\left(\min \left\{\left\|F_{1}-F_{2}\right\|_{\mathbf{D}_{1}^{j}(\mathcal{W}: G)}, 1\right\}\right) .
$$


For $r \geq 2(r \in \mathbf{N})$, we introduce the spaces:

$$
\mathbf{D}_{r}^{p}(\mathcal{W}: G)=\left\{F \in \mathbf{D}_{r-1}^{p}(\mathcal{W}: G): D F \in \mathbf{D}_{r-1}^{p}(\mathcal{W}: H \otimes G)\right\}
$$

with $\|F\|_{\mathbf{D}_{r}^{p}(\mathcal{W}: G)}=\|F\|_{\mathbf{D}_{r-1}^{p}(\mathcal{W}: G)}+\left\|D^{r-1} F\right\|_{\mathbf{D}_{1}^{p}\left(H^{\otimes(r-1) \otimes G)}\right.}$. We also define $\mathbf{D}_{0}^{p}(\mathcal{W}: G)$ as $\mathbf{D}_{0}^{p}(\mathcal{W}: G)=L^{p}(\mathcal{W}:$ $G)$

If $G=\mathbf{R}^{n}$, Then we write $\mathbf{D}_{r}^{p}(\mathcal{W})$ for $\mathbf{D}_{r}^{p}(\mathcal{W}: G)$.

Some properties of these spaces are the following; $\mathbf{D}_{r^{\prime}}^{p^{\prime}}(\mathcal{W}) \subset \mathbf{D}_{r}^{p}(\mathcal{W}), r^{\prime} \leq r$, and $p^{\prime} \leq p$. The dual space of $\left(\mathbf{D}_{r}^{q}(\mathcal{W})\right),\left(\mathbf{D}_{r}^{q}(\mathcal{W})\right)^{*}$ is given by $\left(\mathbf{D}_{r}^{q}(\mathcal{W})\right)^{*}=\mathbf{D}_{-r}^{p}(\mathcal{W})$, with $p^{-1}+q^{-1}=1, r \geq 0$.

Furthermore, define the space $\mathbf{D}_{\infty}(\mathcal{W})=\cap_{p \geq 1, r \geq 0} \mathbf{D}_{r}^{p}(\mathcal{W})$. Then, $\mathbf{D}_{\infty}(\mathcal{W})$ is a complete metric space under a metric,

$$
d_{\mathbf{D}^{\infty}(\mathcal{W})}\left(F_{1}, F_{2}\right)=\sum_{p, r=1}^{\infty} 2^{-p-r}\left(\min \left\{\left\|F_{1}-F_{2}\right\|_{\mathbf{D}_{r}^{p}}, 1\right\}\right) .
$$

We call $F \in \mathbf{D}_{\infty}(\mathcal{W})$ the smooth functional in the sense of Malliavin.

Given $Z=\left(Z_{1}(w), \cdots, Z_{d}(w)\right) \in \mathbf{D}_{1}^{p}(\mathcal{W}: H)$, there exists $D_{i}^{*}\left(Z_{i}\right) \in L^{p}(\mathcal{W}), i=1, \cdots, d$ such that $E\left[\int_{0}^{T} D_{t, i} F(w) Z_{i}(w) d t\right]=E\left[F(w) D_{i}^{*}\left(Z_{i}(w)\right)\right]$ for all $F \in \mathbf{D}_{1}^{\infty-}(\mathcal{W})$. Then, define $D^{*}(Z):=\sum_{i=1}^{d} D_{i}^{*}\left(Z_{i}(w)\right)$. So, there exists $C_{p}>0$ such that $\left\|D^{*}(Z)\right\|_{L^{p}} \leq C_{p}\|Z\|_{\mathbf{D}_{1}^{p}(\mathcal{W}: H)}$. We call $D^{*}(Z)$ the divergence of $Z$.

We also introduce the notation $D_{Z} F$ such that $D_{Z} F:=\sum_{i=1}^{d} \int_{0}^{T} D_{t, i} F(w) Z_{i}(w) d t$. Then, we have $E\left[D_{Z} F\right]=$ $E\left[F D^{*}(Z)\right]$. Thus,

$$
D^{*}(F Z)=F D^{*}(Z)-D_{Z} F
$$

is obtained. (e.g. Proposition 1.16 in Malliavin-Thalmaier (2006))

Definition 2.1 Let $F=\left(F_{1}, \cdots, F_{n}\right) \in \mathbf{D}_{\infty}\left(\mathcal{W}: \mathbf{R}^{n}\right)$ be the $n$-dimensional smooth functional; we call $F$ a nondegenerate in the sense of Malliavin if the Malliavin covariance matrix $\left\{\sigma_{F}^{i j}\right\}_{1 \leq i, j \leq n}$

$$
\sigma_{F}^{i j}:=\left\langle D F_{i}, D F_{j}\right\rangle_{H}=\sum_{k=1}^{d} \int_{0}^{T}\left(D_{t, k} F_{i}(w)\right)\left(D_{t, k} F_{j}(w)\right) d t
$$

is invertible a.s. and

$$
\left(\operatorname{det} \sigma_{F}\right)^{-1} \in L^{\infty-}(\mathcal{W})
$$

Theorem 2.1 Let $F \in \mathbf{D}_{\infty}\left(\mathcal{W}: \mathbf{R}^{n}\right)$ be a n-dimensional nondegenerate in the sense of Malliavin and $G \in \mathbf{D}_{\infty}(\mathcal{W})$. Then, for $\varphi \in C_{b}^{1}\left(\mathbf{R}^{n}\right)$,

$$
E\left[\partial_{i} \varphi(F) G\right]=E\left[\varphi(F) D^{*}\left(\sum_{j=1}^{n} G \gamma_{i j}^{F} D F^{j}\right)\right]
$$

where $\left(\gamma_{i j}^{F}\right)_{1 \leq i, j \leq n}$ is the inverse matrix of Malliavin covariance of $F$.

(Proof) See Lemma III.5.2. of Malliavin (1997).

Theorem 2.2 Let $F \in \mathbf{D}_{\infty}\left(\mathcal{W}: \mathbf{R}^{n}\right)$ be a nondegenerate functional. $F$ has a smooth density $p^{F} \in \mathcal{S}\left(\mathbf{R}^{n}\right)$ where $\mathcal{S}\left(\mathbf{R}^{n}\right)$ denotes the space of all infinitely differentiable functions $f: \mathbf{R}^{n} \mapsto \mathbf{R}$ such that for any $k \geq 1$, and for any multi-index $\beta \in\{1, \cdots, n\}^{j}$ one has $\sup _{x \in \mathbf{R}^{n}}|x|^{k}\left|\partial_{\beta} f(x)\right|<\infty$. (i.e. $\mathcal{S}\left(\mathbf{R}^{n}\right)$ is the Schwartz space and $\mathcal{S}^{\prime}\left(\mathbf{R}^{n}\right)$ is its dual.)

(Proof) See Theorem III.5.1. of Malliavin (1997).

Definition 2.2 Consider the space $\mathbf{D}_{-\infty}(\mathcal{W})=\cup_{p \geq 1, r \geq 0} \mathbf{D}_{-r}^{p}(\mathcal{W})$, that is, the dual of $\mathbf{D}_{\infty}$. We call $F \in \mathbf{D}_{-\infty}(\mathcal{W})$ a distribution on the Wiener space. We define the duality form on $\mathbf{D}_{-\infty} \times \mathbf{D}_{\infty},(F, G) \mapsto\langle F, G\rangle_{\mathbf{D}_{-\infty} \times \mathbf{D}_{\infty}}=$ $E[F G] \in \mathbf{R}$. We call this duality form the generalized expectation.

\subsection{Asymptotic Expansion for Expectation of Generalized Wiener Functionals}

Let $F \in \mathbf{D}_{\infty}\left(\mathcal{W}: \mathbf{R}^{n}\right)$ be a nondegenerate functional, and $\nu$ and $p^{F}$ be the law and the smooth density of $F$, respectively; that is, $\nu(d x)=\mu \circ F^{-1}(d x)=p^{F}(x) d x$. Also, we define the range $O$ as $O:=\left\{x: p^{F}(x)>0\right\} \subset \mathbf{R}^{n}$.

By Malliavin (1997) and Malliavin-Thalmaier (2006), the conditional expectation of $g \in L^{p}(\mathcal{W}, \mu)$ conditioned by a set $\{w: F(w)=x\}$ in $\sigma$-field $\sigma(F), E[g \mid F=x]$ gives a map,

$$
E^{F}: L^{p}(\mathcal{W}, \mu) \ni g \mapsto E[g \mid F=x] \in L^{p}(O, \nu) .
$$

For multi-index $\alpha^{(k)}=\left(\alpha_{1}, \cdots, \alpha_{k}\right)$, we define the iterated Malliavin weight. The Malliavin weight $H_{\alpha(k)}$ is recursively defined as follows: for $G \in \mathbf{D}_{\infty}$,

$$
H_{\alpha^{(k)}}(F, G)=H_{\left(\alpha_{k}\right)}\left(F, H_{\alpha}^{(k-1)}(F, G)\right),
$$


where

$$
H_{(l)}(F, G)=D^{*}\left(\sum_{i=1}^{n} G \gamma_{l i}^{F} D F_{i}\right) .
$$

Here, $\gamma^{F}=\left\{\gamma_{i j}^{F}\right\}_{1 \leq i, j \leq n}$ denotes the inverse matrix of the Malliavin covariance matrix of $F$.

Watanabe $(1983,1984)$ introduced the distribution on Wiener space as composition of a nondegenerate map $F$ by a Schwartz distribution $T$. The next theorem restates the result of Watanabe (1984) in terms of Malliavin (1997) and Malliavin-Thalmaier (2006).

Theorem 2.3 1. Let $\mathcal{S}^{\prime}$ be the Schwartz distributions. There exists a map

$$
\left(E^{F}\right)^{*}: \mathcal{S}^{\prime} \ni T \mapsto T \circ F \in \tilde{\mathbf{D}}_{-\infty}:=\cup_{s \geq 0} \cap_{q \geq 1} \mathbf{D}_{-s}^{q} \subset \mathbf{D}_{-\infty} .
$$

$\left(E^{F}\right)^{*}$ is called the lifting up of $T$.

2. The conditional expectation defines a map

$$
E^{F}: \mathbf{D}_{\infty} \ni G \mapsto E^{F}[G] \in \mathcal{S}(O),
$$

where $\mathcal{S}(O)$ stands for the Schwartz space of the rapidly decreasing functions on $O=\left\{x: p^{F}(x)>0\right\} \subset \mathbf{R}^{n}$. We call this map the push down of $G$.

3. The following duality formula is obtained:

$$
\left\langle\left(E^{F}\right)^{*} T, G\right\rangle_{\mathbf{D}_{-\infty} \times \mathbf{D}_{\infty}}=\left\langle T, E^{F}[G]\right\rangle_{p^{F}(x) d x}
$$

where $\langle\cdot, \cdot\rangle_{p} F(x) d x$ is defined as follows:

$$
\left\langle T, E^{F}[G]\right\rangle_{p^{F}(x) d x}={ }_{\mathcal{S}^{\prime}}\left\langle T, E^{F}[G] p^{F}\right\rangle_{\mathcal{S}} .
$$

(Proof)

In this proof, we apply the discussions of Watanabe (1984), Malliavin (1997), Malliavin-Thalmaier (2006) and Nualart (2006).

1. Given $T \in \mathcal{S}^{\prime}$, there exists $T_{n} \in \mathcal{S}$ such that $T_{n} \rightarrow T$ in $\mathcal{S}^{\prime}$, i.e. for $m \geq 1$,

$$
\left\|A^{-m} T_{n}-A^{-m} T\right\|_{\infty} \rightarrow 0, \quad n \rightarrow \infty,
$$

where $\|f\|_{\infty}=\sup _{x \in \mathbf{R}^{n}}|f(x)|, A=1+|x|^{2}-\Delta, \Delta=\frac{1}{2} \sum_{i=1}^{n} \frac{\partial^{2}}{\partial x_{i}^{2}}$. By the Malliavin integration-by-parts formula, we can estimate as follows; for $p^{-1}+q^{-1}=1$,

$$
\begin{aligned}
\left\|T_{n}(F)-T_{n^{\prime}}(F)\right\|_{\mathbf{D}_{-2 m}^{q}} & =\sup _{G \in \mathbf{D}_{2 m}^{p},\|G\|_{\mathbf{D}_{2 m}^{p}}^{p} \leq 1}\left|E\left[T_{n}(F) G\right]-E\left[T_{n^{\prime}}(F) G\right]\right| \\
& =\sup _{G \in \mathbf{D}_{2 m}^{p},\|G\|_{\mathbf{D}_{2 m}^{p}}^{p} \leq 1}\left|E\left[\left(A^{m}\left\{A^{-m} T_{n}\right\}\right)(F) G\right]-E\left[\left(A^{m}\left\{A^{-m} T_{n^{\prime}}\right\}\right)(F) G\right]\right| \\
& =\sup _{G \in \mathbf{D}_{2 m}^{p},\|G\|_{\mathbf{D}_{2 m}^{p}}^{p} \leq 1}\left|E\left[\left\{A^{-m} T_{n}\right\}(F) \pi_{(2 m)}^{F}(G)\right]-E\left[\left\{A^{-m} T_{n^{\prime}}\right\}(F) \pi_{(2 m)}^{F}(G)\right]\right| \\
& \leq \sup _{G \in \mathbf{D}_{2 m}^{p},\|G\|_{\mathbf{D}_{2 m}^{p}}^{p} \leq 1}\left\|A^{-m} T_{n}-A^{-m} T_{n^{\prime}}\right\|\left\|_{\infty}\right\| \pi_{(2 m)}^{F}(G) \|_{L^{1}} \\
& =C\left\|A^{-m} T_{n}-A^{-m} T_{n^{\prime}}\right\|_{\infty} \rightarrow 0,
\end{aligned}
$$

as $n, n^{\prime} \rightarrow \infty$, where $\pi_{(2 m)}^{F}(G) \in \mathbf{D}_{\infty}$ and $C:=\sup _{G \in \mathbf{D}_{2 m}^{p},\|G\|_{\mathbf{D}_{2 m}^{p}} \leq 1}\left\|\pi_{(2 m)}^{F}(G)\right\|_{L^{1}}<\infty$. Here, as in p.379 of Ikeda-Watanabe (1989) or Theorem 3.2.1 with the equations (3.2.1) and (3.2.2) of Sakamoto-Yoshida (1994), we have used the relation:

$$
E\left[\left(A^{m}\left\{A^{-m} T_{n}\right\}\right)(F) G\right]=E\left[\left\{A^{-m} T_{n}\right\}(F) \pi_{(2 m)}^{F}(G)\right],
$$

where $\pi_{(2 m)}^{F}(G)$ is recursively obtained by

$$
\begin{aligned}
& \pi_{(2)}^{F}(G)=\left(1+|F|^{2}\right) G-1 / 2 \sum_{i=1}^{n} H_{(i, i)}(F, G), \\
& \pi_{(2(m+1))}^{F}(G)=\pi_{(2)}^{F}\left(\pi_{(2 m)}^{F}(G)\right), m \geq 1 .
\end{aligned}
$$

Then $\left(T_{n}(F)\right)_{n \in \mathbf{N}}$ is a Cauchy sequence in $\mathbf{D}_{-\infty}$ and thus there exists $\left(E^{F}\right)^{*} T=T(F) \in \mathbf{D}_{-\infty}$ : a composite functional $T(F)$ is uniquely determined. 
2. Given $G \in \mathbf{D}_{\infty}$. For any multi-index $s=\left(s_{1}, \cdots, s_{k}\right)$, For any $\varphi \in C_{b}^{|s|}\left(\mathbf{R}^{n}\right)$, by push-down and then the integration by parts formula on $\mathbf{R}^{n}$ or the integration by parts formula on $\mathcal{W}$ and then push-down, we obtain

$$
E\left[\partial_{s}^{|s|} \varphi(F) G\right]=(-1)^{|s|} \int_{\mathbf{R}^{n}} \varphi(x) \partial_{s}^{|s|}\left\{E^{F=x}[G] p^{F}(x)\right\} d x=\int_{\mathbf{R}^{n}} \varphi(x) E^{F=x}\left[\pi_{s}^{F}\right] p^{F}(x) d x,
$$

where $\pi_{s}^{F}=H_{s}(F, G) \in \mathbf{D}_{\infty}$. It implies that

$$
(-1)^{|s|} \partial_{s}^{|s|}\left\{E^{F=x}[G] p^{F}(x)\right\}=E^{F=x}\left[\pi_{s}^{F}\right] p^{F}(x),
$$

where

$$
E^{F=x}\left[\pi_{s}^{F}\right] \in L^{p}(O, \nu) .
$$

We define $O_{\epsilon}$ as $O_{\epsilon}=\left\{x \in \mathbf{R}^{n}: p^{F}(x)>\epsilon\right\}$. Therefore,

$$
(-1)^{|s|} \partial_{s}^{|s|}\left\{E^{F=x}[G] p^{F}(x)\right\} \in L^{p}\left(O_{\epsilon}, d x\right),
$$

for all $s$, which implies $E^{F=x}[G] p^{F}(x) \in C^{\infty}(O)$. As $p^{F}(x) \in C^{\infty}(O)$ where $C^{\infty}(O)$ stands for the set of real-valued $C^{\infty}$-functions on $O$,

$$
\left(p^{F}(x)\right)^{-1}\left\{E^{F=x}[G] p^{F}(x)\right\}=E^{F=x}[G] \in C^{\infty}(O) .
$$

Note also that the conditional expectation has the following expression:

$$
E^{F=x}[G] p^{F}(x)=E\left[\mathbf{1}_{\{F>x\}} H_{(1, \cdots, n)}(F, G)\right] .
$$

Thus, for all $k \in \mathbf{N}$ and for all $j=1, \cdots, n$, if $x_{j}>0$,

$$
\begin{aligned}
& \sup _{x \in \mathbf{R}^{d}, x_{j}>0} x_{j}^{2 k} \partial_{s}^{|s|}\left\{E^{F=x}[G] p^{F}(x)\right\} \\
= & \sup _{x \in \mathbf{R}^{d}, x_{j}>0} x_{j}^{2 k} \mid E\left[\mathbf{1}_{F>x} H_{s}\left(F, H_{(1, \cdots, n)}(F, G)\right) \mid\right. \\
\leq & E\left[\left|F_{j}\right|^{2 k}\left|H_{s}\left(F, H_{(1, \cdots, n)}(F, G)\right)\right|\right] \\
< & \infty,
\end{aligned}
$$

if $x_{j}<0$, we can derive a similar estimate. These facts imply $E^{F=x}[G] \in \mathcal{S}(O)$.

3. Hence, because there exists $T_{n} \in \mathcal{S}, n \in \mathbf{N}$ for $T \in \mathcal{S}^{\prime}$ such that $T_{n} \rightarrow T$ in $\mathcal{S}^{\prime}$, we have

$$
\left\langle T_{n}, E^{F}[G]\right\rangle_{p^{F}(x) d x}=E\left[T_{n}(F) G\right] \rightarrow\left\langle\left(E^{F}\right)^{*} T, G\right\rangle_{\mathbf{D}_{-\infty} \times \mathbf{D}_{\infty}}=\left\langle T, E^{F}[G]\right\rangle_{p^{F}(x) d x},
$$

as $n \rightarrow \infty$

Hereafter, we use the notation $\int T(x) p(x) d x$ for $T \in \mathcal{S}^{\prime}\left(\mathbf{R}^{n}\right)$ and $p \in \mathcal{S}\left(\mathbf{R}^{n}\right)$ meaning that $\mathcal{S}^{\prime}\langle T, p\rangle_{\mathcal{S}}$.

The next theorem presents an asymptotic expansion formula for the expectation of generalized Wiener functionals.

Theorem 2.4 Consider a family of smooth nondegenerate Wiener functionals $F^{\epsilon}=\left(F_{1}^{\epsilon}, \cdots, F_{n}^{\epsilon}\right) \in \mathbf{D}_{\infty}\left(\mathcal{W}: \mathbf{R}^{n}\right)$, $\epsilon \in(0,1]$, such that $F^{\epsilon}$ has an asymptotic expansion in $\mathbf{D}_{\infty}$ and satisfies the uniformly nondegenerate condition:

$$
\limsup _{\epsilon \downarrow 0}\left\|\left(\operatorname{det} \sigma_{F^{\epsilon}}\right)^{-1}\right\|_{L^{p}}<\infty, \text { for all } p<\infty .
$$

Then, for a Schwartz distribution $T \in S^{\prime}\left(\mathbf{R}^{n}\right)$, we have an asymptotic expansion in $\mathbf{R}$ :

$$
\left|E\left[T\left(F^{\epsilon}\right)\right]-\left\{\int_{\mathbf{R}^{n}} T(x) p^{F^{0}}(x) d x+\sum_{j=1}^{N} \epsilon^{j} \int_{\mathbf{R}^{n}} T(x) E\left[\sum_{k}^{(j)} H_{\alpha^{(k)}}\left(F^{0}, \prod_{l=1}^{k} F_{\alpha_{l}}^{0, \beta_{l}}\right) \mid F^{0}=x\right] p^{F^{0}}(x) d x\right\}\right|=O\left(\epsilon^{N+1}\right),
$$

where $p^{F^{0}}$ is the density of $F^{0}$, and $F_{i}^{0, k}:=\left.\frac{1}{k !} \frac{d^{k}}{d \epsilon^{k}} F_{i}^{\epsilon}\right|_{\epsilon=0}, k \in \mathbf{N}, i=1, \cdots, n$. Also, $\alpha^{(k)}$ denotes a multi-index, $\alpha^{(k)}=\left(\alpha_{1}, \cdots, \alpha_{k}\right)$ and

$$
\sum_{k}^{(j)} \equiv \sum_{k=1}^{j} \sum_{\beta_{1}+\cdots+\beta_{k}=j, \beta_{i} \geq 1} \sum_{\alpha^{(k)} \in\{1, \cdots, n\}^{k}} \frac{1}{k !}
$$


Moreover, Malliavin weight $H_{\alpha(k)}$ is recursively defined as follows:

$$
H_{\alpha^{(k)}}(F, G)=H_{\left(\alpha_{k}\right)}\left(F, H_{\alpha^{(k-1)}}(F, G)\right),
$$

where

$$
H_{(l)}(F, G)=D^{*}\left(\sum_{i=1}^{n} G \gamma_{l i}^{F} D F_{i}\right) .
$$

Here, $\gamma^{F}=\left\{\gamma_{i j}^{F}\right\}_{1 \leq i, j \leq n}$ denotes the inverse matrix of the Malliavin covariance matrix of $F$.

(Proof)

We use $\alpha$ as an abbreviation of $\alpha^{(k)}$ in the proof. Under the uniformly non-degenerate condition of $F^{\epsilon} \in$ $\mathbf{D}_{\infty}\left(\mathcal{W}: \mathbf{R}^{n}\right)$, the lifting up of $T \in \mathcal{S}^{\prime}\left(\mathbf{R}^{n}\right),\left(E^{F^{\epsilon}}\right)^{*} T$, has the asymptotic expansion in distributions on the Wiener space $\mathbf{D}_{-\infty}$, i.e. for $N \in \mathbf{N}$, there exists $s \in \mathbf{N}$ s.t.

$$
\left\|\left(E^{F^{\epsilon}}\right)^{*} T-\left\{T \circ F^{0}+\sum_{j=1}^{N} \epsilon^{j} \sum_{k}^{(j)}\left(\partial_{\alpha}^{k} T\right) \circ F^{0} \prod_{l=1}^{k} F_{\alpha_{l}}^{0, \beta_{l}}\right\}\right\|_{\mathbf{D}_{-s}^{q}}=O\left(\epsilon^{N+1}\right), \quad \epsilon \in(0,1], q<\infty
$$

Then, there exists an asymptotic expansion of $\left\langle\left(E^{F^{\epsilon}}\right)^{*} T, \mathbf{1}\right\rangle_{\mathbf{D}_{-\infty} \times \mathbf{D}_{\infty}}$. The push-down of the Malliavin weights are computed as follows:

$$
\begin{aligned}
& \left\langle\partial_{\alpha}^{k} T\left(F^{0}\right), \prod_{l=1}^{k} F_{\alpha_{l}}^{0, \beta_{l}}\right\rangle_{\mathbf{D}_{-\infty} \times \mathbf{D}_{\infty}}=\left\langle T\left(F^{0}\right), H_{\alpha}\left(F^{0}, \prod_{l=1}^{k} F_{\alpha_{l}}^{0, \beta_{l}}\right)\right\rangle_{\mathbf{D}_{-\infty} \times \mathbf{D}_{\infty}} \\
& =\left\langle T, E^{F^{0}}\left[H_{\alpha}\left(F^{0}, \prod_{l=1}^{k} F_{\alpha_{l}}^{0, \beta_{l}}\right)\right]\right\rangle_{p^{F^{0}}(x) d x} \\
& =\int_{\mathbf{R}^{n}} T(x) E\left[H_{\alpha}\left(F^{0}, \prod_{l=1}^{k} F_{\alpha_{l}}^{0, \beta_{l}}\right) \mid F^{0}=x\right] p^{F^{0}}(x) d x .
\end{aligned}
$$

Corollary 2.1 The density $p^{F^{\epsilon}}(y)$ is expressed as following asymptotic expansion with the push-down of Malliavin weights:

$$
p^{F^{\epsilon}}(y)=p^{F^{0}}(y)+\sum_{j=1}^{N} \epsilon^{j} E\left[\sum_{k}^{(j)} H_{\alpha}(k)\left(F^{0}, \prod_{l=1}^{k} F_{\alpha_{l}}^{0, \beta_{l}}\right) \mid F^{0}=y\right] p^{F^{0}}(y)+O\left(\epsilon^{N+1}\right),
$$

where $p^{F^{0}}(y)$ is the density of $F^{0}$.

(Proof)

Take a delta function $\delta_{y} \in \mathcal{S}^{\prime}$ in the theorem above.

\section{Asymptotic Expansion in Multi-Dimensional Stochastic Volatil- ity Model}

This section applies the general formula in the previous section to pricing options in a stochastic volatility model and then obtains a new implied volatility's expansion formula.

\subsection{Asymptotic Expansion of Option Prices}

This subsection proves a basic result on an asymptotic expansion for a stochastic volatility model.

Let $\left(\Omega, \mathcal{F},\left(\mathcal{F}_{t}\right)_{t \in[0, T]}, P\right)$ be a filtered probability space and $W=\left\{\left(W_{1, t}, \cdots, W_{d, t}\right): 0 \leq t \leq T\right\}$ be a $d$ dimensional Brownian motion with respect to $\left(\mathcal{F}_{t}\right)_{t \in[0, T]}$. We consider the following stochastic volatility model (n-dimensional volatility factor).

$$
\begin{aligned}
& d S_{t}^{(\epsilon)}=r S_{t}^{(\epsilon)} d t+V\left(\sigma_{t}^{(\epsilon)}\right) S_{t}^{(\epsilon)} d W_{1, t}, \\
& d \sigma_{t}^{(\epsilon)}=A_{0}\left(\sigma_{t}^{(\epsilon)}\right) d t+\epsilon A\left(\sigma_{t}^{(\epsilon)}\right) d W_{t}, \\
& S_{0}^{(\epsilon)}=S_{0}^{(0)}=s>0, \sigma_{0}^{(\epsilon)}=\sigma_{0}^{(0)}=\sigma \in \mathbf{R}_{+}^{n},
\end{aligned}
$$


where $V \in C_{b}^{\infty}\left(\mathbf{R}^{n} \rightarrow \mathbf{R}\right), A_{0} \in C_{b}^{\infty}\left(\mathbf{R}^{n} \rightarrow \mathbf{R}^{n}\right), A \in C_{b}^{\infty}\left(\mathbf{R}^{n} \rightarrow \mathbf{R}^{n \times d}\right), r>0$ and $\epsilon \in[0,1]$. Note that $\epsilon$ is the volatility of volatility parameter. $\left(\sigma_{t}^{(0)}\right)_{t \in[0, T]}$ is a deterministic process and satisfies an ordinary differential equation,

$$
d \sigma_{t}^{(0)}=A_{0}\left(\sigma_{t}^{(0)}\right) d t
$$

Next, we impose the following condition which gives the nondegeneracy of the Malliavin covariance of $S_{T}^{(\epsilon)}$ at $\epsilon=0$.

Assumption 3.1 For some $s \in[0, T], V\left(\sigma_{s}^{(0)}\right) \neq 0$.

We define the logarithmic process of $\left(S_{t}^{(\epsilon)}\right)_{t \in[0, T]}$ as

$$
X_{t}^{(\epsilon)}=\log \left(\frac{S_{t}^{(\epsilon)}}{s}\right) .
$$

Let $p^{S V}(y)$ be the density of the underlying asset of the stochastic volatility model and $C^{S V}(T, K)$ and $P^{S V}(T, K)$ be the call and the put option prices under the stochastic volatility with maturity $T$ and strike price $K$.

Also let $p^{B S}(y)$ is the log-normal density of the Black-Scholes model, i.e.

$$
p^{B S}(y):=\frac{1}{y \sqrt{2 \pi \int_{0}^{T} V\left(\sigma_{t}^{(0)}\right)^{2} d t}} \exp \left(-\frac{1}{2 \int_{0}^{T} V\left(\sigma_{t}^{(0)}\right)^{2} d t}\left(\log \left(\frac{y}{s}\right)-r T+\frac{1}{2} \int_{0}^{T} V\left(\sigma_{t}^{(0)}\right)^{2} d t\right)^{2}\right) .
$$

$C^{B S}(T, K, \bar{\sigma})$ and $P^{B S}(T, K, \bar{\sigma})$ denote the Black-Scholes formula of the call and the put options with maturity $T$ and strike price $K$, i.e.

$$
\begin{aligned}
& C^{B S}(T, K, \bar{\sigma}):=s N\left(d_{1}\right)-K e^{-r T} N\left(d_{2}\right), \\
& P^{B S}(T, K, \bar{\sigma}):=K e^{-r T} N\left(-d_{2}\right)-s N\left(-d_{1}\right),
\end{aligned}
$$

where

$$
\begin{aligned}
n(x) & :=\frac{1}{\sqrt{2 \pi}} e^{-\frac{x^{2}}{2}}, \\
N(x) & :=\int_{-\infty}^{x} n(y) d y, \\
d_{1} & :=\frac{\log \left(\frac{s}{K}\right)+r T}{\bar{\sigma} \sqrt{T}}+\frac{1}{2} \bar{\sigma} \sqrt{T}, \\
d_{2} & :=\frac{\log \left(\frac{s}{K}\right)+r T}{\bar{\sigma} \sqrt{T}}-\frac{1}{2} \bar{\sigma} \sqrt{T},
\end{aligned}
$$

with

$$
\bar{\sigma}:=\left(\frac{1}{T} \int_{0}^{T} V\left(\sigma_{t}^{(0)}\right)^{2} d t\right)^{1 / 2}
$$

Let $\sigma_{S_{T}^{(0)}}$ be the Malliavin (co)variance of $S_{T}^{(\epsilon)}$ at $\epsilon=0$, i.e.

$$
\sigma_{S_{T}^{(0)}}=\left\|D_{1} S_{T}^{(0)}\right\|_{H}^{2}=\left(S_{T}^{(0)}\right)^{2} \int_{0}^{T} V\left(\sigma_{s}^{(0)}\right)^{2} d s
$$

We introduce the expressions;

$$
\begin{aligned}
Z(t) & :=\frac{D_{t, 1} S_{T}^{(0)}}{\left\|D_{1} S_{T}^{(0)}\right\|_{H}^{2}}=\frac{S_{T}^{(0)} V\left(\sigma_{t}^{(0)}\right)}{\left(S_{T}^{(0)}\right)^{2} \int_{0}^{T} V\left(\sigma_{u}^{(0)}\right)^{2} d u}, \\
S_{t}^{(k)} & =\left.\frac{1}{k !} \frac{\partial^{k}}{\partial \epsilon^{k}} S_{t}^{(\epsilon)}\right|_{\epsilon=0}, \\
\Psi^{\beta_{1}, \cdots, \beta_{k}}(t) & =\prod_{i=1}^{k} S_{t}^{\left(\beta_{i}\right)}
\end{aligned}
$$

where $\beta_{l} \geq 1$ satisfy

$$
\sum_{l=1}^{k} \beta_{l}=j, \quad j \in \mathbf{N}, \quad 1 \leq k \leq j .
$$

Given $Z$ above, for $\Psi \in \mathbf{D}_{\infty}, D^{*}(Z)-D_{Z}: \mathbf{D}_{\infty} \rightarrow \mathbf{D}_{\infty}$ is expressed as

$$
\left(D^{*}(Z)-D_{Z}\right) \circ \Psi=\Psi \int_{0}^{T} Z(t) d W_{1, t}-\int_{0}^{T} D_{t, 1} \Psi Z(t) d t .
$$

Then, we have the following result. 
Theorem 3.1 Under the stochastic volatility model (28), we have asymptotic expansions of the density and the option prices as follows:

$$
\begin{aligned}
p^{S V}(y) & =p^{B S}(y)+\sum_{j=1}^{N} \epsilon^{j} E\left[\pi_{j} \mid S_{T}^{(0)}=y\right] p^{B S}(y)+O\left(\epsilon^{N+1}\right), \\
C^{S V}(T, K) & =C^{B S}(T, K, \bar{\sigma})+\sum_{j=1}^{N} \epsilon^{j} \int_{\mathbf{R}_{+}} e^{-r T}(y-K)^{+} E\left[\pi_{j} \mid S_{T}^{(0)}=y\right] p^{B S}(y) d y+O\left(\epsilon^{N+1}\right), \\
P^{S V}(T, K) & =P^{B S}(T, K, \bar{\sigma})+\sum_{j=1}^{N} \epsilon^{j} \int_{\mathbf{R}_{+}} e^{-r T}(K-y)^{+} E\left[\pi_{j} \mid S_{T}^{(0)}=y\right] p^{B S}(y) d y+O\left(\epsilon^{N+1}\right),
\end{aligned}
$$

where $\pi_{j}$ is the $j^{\text {th }}$-order Malliavin weight, i.e.

$$
\pi_{j}=\sum_{k=1}^{j} \sum_{\beta_{1}+\cdots+\beta_{k}=j, \beta_{i} \geq 1} \frac{1}{k !} H_{k}\left(S_{T}^{(0)}, \Psi^{\beta_{1}, \cdots, \beta_{k}}(T)\right) \in \mathbf{D}_{\infty}
$$

with

$$
\begin{aligned}
& H_{1}\left(S_{T}^{(0)}, \Psi^{\beta_{1}, \cdots, \beta_{k}}(T)\right)=\Psi^{\beta_{1}, \cdots, \beta_{k}}(T) \int_{0}^{T} Z(s) d W_{1, s}-\int_{0}^{T} D_{s, 1} \Psi^{\beta_{1}, \cdots, \beta_{k}}(T) Z(s) d s, \\
& H_{k}\left(S_{T}^{(0)}, \Psi^{\beta_{1}, \cdots, \beta_{k}}(T)\right)=\left(D^{*}(Z)-D_{Z}\right)^{k} \circ \Psi^{\beta_{1}, \cdots, \beta_{k}} .
\end{aligned}
$$

(Proof)

Let $\left(Y_{t}\right)_{t}$ be the solution of the following stochastic differential equation;

$$
\begin{aligned}
d Y_{j}^{i}(t) & =\sum_{l=1}^{d} \sum_{k=1}^{n} a_{k, l}^{i}(s) Y_{j}^{k}(s) d W_{t}^{l}+\sum_{k=1}^{n} b_{k}^{i}(s) Y_{j}^{k}(s) d s, \\
Y_{j}^{i}(0) & =\delta_{j}^{i},
\end{aligned}
$$

where

$$
\begin{aligned}
a_{k, l}^{i}(s) & =\partial_{k} A_{l}^{i}\left(\sigma_{s}^{(\epsilon)}\right), \\
b_{k}^{i}(s) & =\partial_{k} A_{0}^{i}\left(\sigma_{s}^{(\epsilon)}\right),
\end{aligned}
$$

and $\delta_{j}^{i}$ is the Kronecker's delta. Let $D_{s, k}, k=1, \cdots, d$ be the Malliavin derivative acting on the Brownian motion $W_{k, t}$. Then, the Malliavin covariance of $S_{T}^{(\epsilon)}$ is given by;

$$
\begin{aligned}
\sigma_{S_{T}^{(\epsilon)}} & =\sum_{i=1}^{d} \int_{0}^{T}\left(D_{s, i} S_{T}^{(\epsilon)}\right)^{2} d s \\
& =\left(S_{T}^{(\epsilon)}\right)^{2} \sum_{i=1}^{d} \int_{0}^{T}\left(D_{s, i} X_{T}^{(\epsilon)}\right)^{2} d s
\end{aligned}
$$

where

$$
\begin{aligned}
D_{s, 1} X_{T}^{(\epsilon)} & =V\left(\sigma_{s}^{(\epsilon)}\right)-\epsilon \sum_{i=1}^{n} \int_{s}^{T} V\left(\sigma_{u}^{(\epsilon)}\right) \partial_{i} V\left(\sigma_{u}^{(\epsilon)}\right) D_{s, 1} \sigma_{i, u}^{(\epsilon)} d u \\
& +\epsilon \sum_{i=1}^{n} \int_{s}^{T} \partial_{i} V\left(\sigma_{u}^{(\epsilon)}\right) D_{s, 1} \sigma_{i, u}^{(\epsilon)} d W_{1, u} \\
D_{s, k} X_{T}^{(\epsilon)} & =-\epsilon \sum_{i=1}^{n} \int_{s}^{T} V\left(\sigma_{u}^{(\epsilon)}\right) \partial_{i} V\left(\sigma_{u}^{(\epsilon)}\right) D_{s, k} \sigma_{i, u}^{(\epsilon)} d u \\
& +\epsilon \sum_{i=1}^{n} \int_{s}^{T} \partial_{i} V\left(\sigma_{u}^{(\epsilon)}\right) D_{s, k} \sigma_{i, u}^{(\epsilon)} d W_{1, u} .
\end{aligned}
$$

Here, $\partial_{i} V\left(\sigma_{u}^{(\epsilon)}\right)=\left.\frac{\partial V(x)}{\partial x_{i}}\right|_{x=\sigma_{u}^{(\epsilon)}}$ and for $s \leq u$,

$$
D_{s, k} \sigma_{i, u}^{(\epsilon)}=\sum_{l, j=1}^{n} Y_{l}^{i}(u) Y^{-1}(s)_{j}^{l} A_{k}^{j}\left(\sigma_{s}^{(\epsilon)}\right)
$$


Assumption 3.1 yields the nondegeneracy of $S_{T}^{(0)}$;

$$
\left\|\sigma_{S_{T}^{(0)}}^{-1}\right\|_{L^{p}}<\infty, \text { for all } p<\infty
$$

Then, the similar argument to Takahashi-Yoshida (2004), we have an asymptotic expansion in $\mathbf{D}_{-\infty}$;

$$
\begin{aligned}
\delta_{y}\left(S_{T}^{(\epsilon)}\right) & =\delta_{y}\left(S_{T}^{(0)}\right)+\sum_{i=1}^{N} \epsilon^{j} \sum_{k=1}^{j} \sum_{\beta_{1}+\cdots+\beta_{k}=j, \beta_{i} \geq 1} \frac{1}{k !} \partial^{k} \delta_{y}\left(S_{T}^{(0)}\right) \Psi^{\beta_{1}, \cdots, \beta_{k}}(T)+O\left(\epsilon^{N+1}\right), \\
e^{-r T}\left(S_{T}^{(\epsilon)}-K\right)^{+} & =e^{-r T}\left(S_{T}^{(0)}-K\right)^{+}+\sum_{i=1}^{N} \epsilon^{j} \sum_{k=1}^{j} \sum_{\beta_{1}+\cdots+\beta_{k}=j, \beta_{i} \geq 1} \frac{1}{k !} e^{-r T} \partial^{k}\left(S_{T}^{(0)}-K\right)^{+} \Psi^{\beta_{1}, \cdots, \beta_{k}}(T)+O\left(\epsilon^{N+1}\right) .
\end{aligned}
$$

By the integration by parts formula,

$$
\begin{aligned}
\left\langle\partial^{k} \delta_{y}\left(S_{T}^{(0)}\right), \Psi^{\beta_{1}, \cdots, \beta_{k}}(T)\right\rangle_{\mathbf{D}_{-\infty} \times \mathbf{D}_{\infty}} & =\left\langle\delta_{y}\left(S_{T}^{(0)}\right), H_{k}\left(S_{T}^{(0)}, \Psi^{\beta_{1}, \cdots, \beta_{k}}(T)\right)\right\rangle_{\mathbf{D}_{-\infty} \times \mathbf{D}_{\infty}} \\
& =\left\langle\delta_{y}, E_{T}^{S_{T}^{(0)}}\left[H_{k}\left(S_{T}^{(0)}, \Psi^{\beta_{1}, \cdots, \beta_{k}}(T)\right)\right]\right\rangle_{p^{B S}(x) d x} \\
& =E\left[H_{k}\left(S_{T}^{(0)}, \Psi^{\beta_{1}, \cdots, \beta_{k}}(T)\right) \mid S_{T}^{(0)}=y\right] p^{B S}(y),
\end{aligned}
$$

where

Similarly, we have

$$
\partial^{k} \delta_{y}\left(S_{T}^{(0)}\right)=\left.\frac{\partial^{k}}{\partial x^{k}} \delta_{y}(x)\right|_{x=S_{T}^{(0)}}
$$

$$
\left\langle e^{-r T} \partial^{k}\left(S_{T}^{(0)}-K\right)^{+}, \Psi^{\beta_{1}, \cdots, \beta_{k}}(T)\right\rangle_{\mathbf{D}_{-\infty} \times \mathbf{D}_{\infty}}=\int_{\mathbf{R}_{+}} e^{-r T}(y-K)^{+} E\left[H_{k}\left(S_{T}^{(0)}, \Psi^{\beta_{1}, \cdots, \beta_{k}}(T)\right) \mid S_{T}^{(0)}=y\right] p^{B S}(y) d y,
$$

where

$$
\partial^{k}\left(S_{T}^{(0)}-K\right)^{+}=\left.\frac{\partial^{k}}{\partial x^{k}}(x-K)^{+}\right|_{x=S_{T}^{(0)}}
$$

Then, we obtain asymptotic expansion formulas of the density and the option prices;

$$
\begin{aligned}
p^{S V}(y) & =p^{B S}(y)+\sum_{j=1}^{N} \epsilon^{j} E\left[\pi_{j} \mid S_{T}^{(0)}=y\right] p^{B S}(y)+O\left(\epsilon^{N+1}\right), \\
C^{S V}(T, K) & =C^{B S}(T, K, \bar{\sigma})+\sum_{j=1}^{N} \epsilon^{j} \int_{\mathbf{R}_{+}} e^{-r T}(y-K)^{+} E\left[\pi_{j} \mid S_{T}^{(0)}=y\right] p^{B S}(y) d y+O\left(\epsilon^{N+1}\right), \\
P^{S V}(T, K) & =P^{B S}(T, K, \bar{\sigma})+\sum_{j=1}^{N} \epsilon^{j} \int_{\mathbf{R}_{+}} e^{-r T}(K-y)^{+} E\left[\pi_{j} \mid S_{T}^{(0)}=y\right] p^{B S}(y) d y+O\left(\epsilon^{N+1}\right) .
\end{aligned}
$$

The Malliavin weights are computed by the iterated Skorohod integrals.

$$
\begin{aligned}
H_{1}\left(S_{T}^{(0)}, \Psi^{\beta_{1}, \cdots, \beta_{k}}\right) & =D^{*}\left(\Psi^{\beta_{1}, \cdots, \beta_{k}} \sigma_{S_{T}^{(0)}}^{-1} D S_{T}^{(0)}\right) \\
& =\left(D^{*}(Z)-D_{Z}\right) \circ \Psi^{\beta_{1}, \cdots, \beta_{k}} \\
& =\Psi^{\beta_{1}, \cdots, \beta_{k}}(T) \int_{0}^{T} Z(s) d W_{1, s}-\int_{0}^{T} D_{s, 1} \Psi^{\beta_{1}, \cdots, \beta_{k}}(T) Z(s) d s \\
H_{k}\left(S_{T}^{(0)}, \Psi^{\beta_{1}, \cdots, \beta_{k}}(T)\right) & =H_{1}\left(S_{T}^{(0)}, H_{k-1}\left(S_{T}^{(0)}, \Psi^{\beta_{1}, \cdots, \beta_{k}}(T)\right)\right) \\
& =\left(D^{*}(Z)-D_{Z}\right)^{k} \circ \Psi^{\beta_{1}, \cdots, \beta_{k}} .
\end{aligned}
$$

\subsection{Implied Volatility Expansion}

This subsection derives an asymptotic expansion formula for the implied volatility in the stochastic volatility model considered in the previous subsection, where we obtained an approximation formula of a call option:

$$
C^{S V}(T, K)=C^{B S}(T, K, \bar{\sigma})+\epsilon C_{1}+\epsilon^{2} C_{2}+\epsilon^{3} C_{3}+O\left(\epsilon^{4}\right)
$$


where

$$
C_{i}=\int_{\mathbf{R}} e^{-r T}\left(s e^{y+r T-\frac{1}{2} \Sigma}-K\right)^{+} E\left[\pi_{i} \mid \int_{0}^{T} V\left(\sigma_{t}^{(0)}\right) d W_{1, t}=y\right] \frac{1}{\sqrt{2 \pi \Sigma}} e^{-\frac{1}{2 \Sigma} y^{2}} d y, \quad i=1,2,3 .
$$

We obtain an asymptotic expansion formula of the implied volatility around $\bar{\sigma}=\left(\frac{1}{T} \int_{0}^{T} V\left(\sigma_{t}^{(0)}\right)^{2} d t\right)^{1 / 2}$.

Theorem 3.2 Under the stochastic volatility model (28), an asymptotic expansion of the implied volatility is given by

$$
\begin{aligned}
& \sigma^{I V}(T, K)=\bar{\sigma}+\epsilon \frac{C_{1}}{C_{\sigma}^{B S}(\bar{\sigma})}+\epsilon^{2}\left\{\frac{C_{2}}{C_{\sigma}^{B S}(\bar{\sigma})}-\frac{1}{2} \frac{C_{1}^{2}}{C_{\sigma}^{B S}(\bar{\sigma})^{3}} C_{\sigma \sigma}^{B S}(\bar{\sigma})\right\} \\
& +\epsilon^{3}\left\{\frac{C_{3}}{C_{\sigma}^{B S}(\bar{\sigma})}-\left(\frac{C_{1}}{C_{\sigma}^{B S}(\bar{\sigma})^{2}}\right)\left\{\frac{C_{2}}{C_{\sigma}^{B S}(\bar{\sigma})}-\frac{1}{2} \frac{C_{1}^{2}}{C_{\sigma}^{B S}(\bar{\sigma})^{3}} C_{\sigma \sigma}^{B S}(\bar{\sigma})\right\} C_{\sigma \sigma}^{B S}(\bar{\sigma})-\frac{1}{3 !} \frac{C_{1}^{3}}{C_{\sigma}^{B S}(\bar{\sigma})^{4}} C_{\sigma \sigma \sigma}^{B S}(\bar{\sigma})\right\}+O\left(\epsilon^{4}\right),
\end{aligned}
$$

where $C_{\sigma}^{B S}(\bar{\sigma}), C_{\sigma \sigma}^{B S}(\bar{\sigma}), C_{\sigma \sigma \sigma}^{B S}(\bar{\sigma})$ are derivatives of the Black-Scholes formula with respect to the volatility, i.e.

$$
\begin{aligned}
C_{\sigma}^{B S}(\bar{\sigma}) & :=\left.\frac{\partial}{\partial \sigma} C^{B S}\right|_{\sigma=\bar{\sigma}}=s \sqrt{T} n\left(d_{1}\right), \\
C_{\sigma \sigma}^{B S}(\bar{\sigma}) & :=\left.\frac{\partial^{2}}{\partial \sigma^{2}} C^{B S}\right|_{\sigma=\bar{\sigma}}=\frac{s \sqrt{T}}{\bar{\sigma}} n\left(d_{1}\right) d_{1} d_{2}, \\
C_{\sigma \sigma \sigma}^{B S}(\bar{\sigma}) & :=\left.\frac{\partial^{3}}{\partial \sigma^{3}} C^{B S}\right|_{\sigma=\bar{\sigma}}=\frac{s \sqrt{T}}{\bar{\sigma}^{2}} n\left(d_{1}\right)\left\{d_{1}^{2} d_{2}^{2}-d_{1} d_{2}-d_{1}^{2}-d_{2}^{2}\right\}
\end{aligned}
$$

(Proof)

Suppose that an implied volatility is expanded as;

$$
\sigma^{I V}(T, K)=\bar{\sigma}+\epsilon \sigma_{1}+\epsilon^{2} \sigma_{2}+\epsilon^{3} \sigma_{3}+O\left(\epsilon^{4}\right) .
$$

Then we have

$$
\begin{aligned}
C^{B S}\left(T, K, \sigma^{I V}(T, K)\right) & =C^{B S}(T, K, \bar{\sigma})+\epsilon C_{\sigma}^{B S}(\bar{\sigma}) \sigma_{1}+\epsilon^{2}\left\{C_{\sigma}^{B S}(\bar{\sigma}) \sigma_{2}+\frac{1}{2} C_{\sigma \sigma}^{B S}(\bar{\sigma})\left(\sigma_{1}\right)^{2}\right\} \\
& +\epsilon^{3}\left\{\sigma_{3} C_{\sigma}^{B S}(\bar{\sigma})+\sigma_{1} \sigma_{2} C_{\sigma \sigma}^{B S}(\bar{\sigma})+\frac{1}{3 !} \sigma_{1}^{3} C_{\sigma \sigma \sigma}^{B S}(\bar{\sigma})\right\}+O\left(\epsilon^{4}\right) .
\end{aligned}
$$

By the definition of the implied volatility in the stochastic volatility, i.e. $C^{S V}(T, K)=C^{B S}\left(T, K, \sigma^{I V}(T, K)\right)$, the approximation terms of the implied volatility are given by

$$
\begin{aligned}
\sigma_{1} & =\frac{C_{1}}{C_{\sigma}^{B S}(\bar{\sigma})}, \\
\sigma_{2} & =\left\{\frac{C_{2}}{C_{\sigma}^{B S}(\bar{\sigma})}-\frac{1}{2} \frac{C_{1}^{2}}{C_{\sigma}^{B S}(\bar{\sigma})^{3}} C_{\sigma \sigma}^{B S}(\bar{\sigma})\right\} \\
\sigma_{3} & =\frac{C_{3}}{C_{\sigma}^{B S}(\bar{\sigma})}-\left(\frac{C_{1}}{C_{\sigma}^{B S}(\bar{\sigma})^{2}}\right)\left\{\frac{C_{2}}{C_{\sigma}^{B S}(\bar{\sigma})}-\frac{1}{2} \frac{C_{1}^{2}}{C_{\sigma}^{B S}(\bar{\sigma})^{3}} C_{\sigma \sigma}^{B S}(\bar{\sigma})\right\} C_{\sigma \sigma}^{B S}(\bar{\sigma})-\frac{1}{3 !} \frac{C_{1}^{3}}{C_{\sigma}^{B S}(\bar{\sigma})^{4}} C_{\sigma \sigma \sigma}^{B S}(\bar{\sigma}) .
\end{aligned}
$$

\subsection{Comments on Related Works}

Fournié et al. (1999) applied Malliavin calculus to efficient Monte Carlo estimators for computing Greeks of options in the Black-Scholes framework, e.g. for $d S_{t}=r S_{t} d t+\sigma^{(\epsilon)} S_{t} d W_{t}$ and $\sigma^{(\epsilon)}=\sigma+\epsilon$,

$$
\frac{\partial}{\partial \sigma} E\left[e^{-r T}\left(S_{T}-K\right)^{+}\right]=\left.\frac{\partial}{\partial \epsilon} E\left[e^{-r T}\left(S_{T}^{(\epsilon)}-K\right)^{+}\right]\right|_{\epsilon=0}=E\left[e^{-r T}\left(S_{T}^{(0)}-K\right)^{+} \pi\right] .
$$

The estimator $\pi$ is the so-called Malliavin weight. Subsequently, a number of papers extended the method. In particular, related to our work, Siopacha and Teichmann (2010) developed strong and weak Taylor methods for a system of stochastic differential equations (SDEs) with perturbations. Especially, the weak Taylor method is based on the integration by parts on the Wiener space, which is a powerful tool for efficient Monte Carlo simulations and is general enough to be applied to multi-dimensional SDEs. As an example, they applied the method to swaptions under a market model of interest rates with one-dimensional Heston-type stochastic volatility and obtained an approximation of the option prices including the expectation of the divergence on the Wiener space. In the last step, they used Monte Carlo simulations for computing the option prices and demonstrated the efficiency of their method comparing with well-known existing Monte Carlo schemes. 
Lewis (2000) developed a new method for approximations of European option prices and implied volatilities. In particular, he proposed an expansion with respect to a stochastic volatility parameter, "vol-of-vol " ( $\xi$ below) under a general one-dimensional time-homogeneous diffusion process of the stochastic volatility, which is considered as a special case of our setup. However, he took a different approach from ours to obtain an approximation: for example, he considered the following generalized Heston model :

$$
\begin{aligned}
d S_{t} & =r S_{t} d t+\sqrt{v_{t}} S_{t} d W_{1 t}, \\
d v_{t} & =b\left(v_{t}\right) d t+\xi \eta\left(v_{t}\right)\left(\rho(v) d W_{1 t}+\sqrt{1-\rho(v)^{2}} d W_{2 t}\right),
\end{aligned}
$$

where $r$ and $\xi$ are constants. Also, $W=\left(W_{1 t}, W_{2 t}\right)$ is a two-dimensional Brownian motion and $\rho(v)$ stands for the instantaneous correlation between $S$ and $v$. For valuation of call options, he used the following formula:

$$
C(T, K)=S_{0}-\frac{K e^{-r T}}{2 \pi} \int_{\frac{i}{2}-\infty}^{\frac{i}{2}+\infty} e^{-i k x} \frac{H(k, v, T)}{k^{2}-i k} d k,
$$

where $x=\log \left(S / K e^{-r T}\right)$, and $H$ ( called "the fundamental transform") is the solution to

$$
\frac{\partial H}{\partial T}=\frac{1}{2} \xi^{2} \eta^{2}(v) \frac{\partial^{2} H}{\partial v^{2}}+(b(v)-i k \xi \rho(v) \eta(v) \sqrt{v}) \frac{\partial H}{\partial v}-\left\{\left(k^{2}-i k\right) / 2\right\} v H,
$$

with the initial condition $H(k, V, 0)=1$. Then, he gave an approximation for a call price showing a scheme for the expansion of $H$ with respect to $\xi$ :

$$
H(k, v, T)=H^{(0)}(k, v, T)+\xi H^{(1)}(k, v, T)+\xi^{2} H^{(2)}(k, v, T)+\cdots .
$$

He also presented an explicit result of the expansion for the following model.

$$
d v_{t}=\left(\omega-\theta v_{t}\right) d t+\xi v_{t}^{\beta}\left(\rho d W_{1 t}+\sqrt{1-\rho^{2}} d W_{2 t}\right),
$$

where $\omega, \theta, \beta$ and $\rho$ are some appropriate constants.

This paper applies an asymptotic expansion approach to stochastic volatility models and derive the approximation terms including the Malliavin weights. Then, these weights are transformed into a finite-dimensional integration through the duality formula of Malliavin (1997); that is, for $T \in \mathcal{S}^{\prime}$,

$$
E\left[T\left(S_{T}\right) \pi\right]=\left\langle\left(E^{S_{T}}\right)^{*} T, \pi\right\rangle_{\mathbf{D}_{-\infty} \times \mathbf{D}_{\infty}}=\left\langle T, E^{S_{T}}[\pi]\right\rangle_{p(x) d x}=\int_{\mathbf{R}} T(x) E\left[\pi \mid S_{T}=x\right] p(x) d x,
$$

where $p(x)$ is the density of $S_{T} \in \mathbf{D}_{\infty}$. This formula suggests that an element of the distributions on the Wiener space $\mathbf{D}_{-\infty}$ is the adjoint operator of the conditional expectation. It also shows that the push-down of the Malliavin weights (the conditional expectation of the divergence on the Wiener space) is an element of the Schwartz space $\mathcal{S}$. Thus, applying both the integration by parts and the duality formula, we can obtain analytical approximations for density functions, option prices and implied volatilities.

As a simple example, for $d S_{t}=r S_{t} d t+\sigma_{t}^{(\epsilon)} S_{t} d W_{1 t}$ and $d \sigma_{t}^{(\epsilon)}=A_{0}\left(\sigma_{t}^{(\epsilon)}\right) d t+\epsilon A_{1}\left(\sigma^{(\epsilon)}\right)\left(\rho d W_{1 t}+\sqrt{1-\rho^{2}} d W_{2 t}\right)$ for some real-valued functions $A_{0}(x)$ and $A_{1}(x)$, we can obtain an approximation for a call option as follows:

$$
\begin{aligned}
C(T, K)=E\left[e^{-r T}\left(S_{T}^{(\epsilon)}-K\right)^{+}\right] & =E\left[e^{-r T}\left(S_{T}^{(0)}-K\right)^{+}\right]+\left.\epsilon \frac{\partial}{\partial \epsilon} E\left[e^{-r T}\left(S_{T}^{(\epsilon)}-K\right)^{+}\right]\right|_{\epsilon=0}+O\left(\epsilon^{2}\right) \\
& =E\left[e^{-r T}\left(S_{T}^{(0)}-K\right)^{+}\right]+\epsilon E\left[e^{-r T}\left(S_{T}^{(0)}-K\right)^{+} \pi\right]+O\left(\epsilon^{2}\right) \\
& =C^{B S}(T, K)+\epsilon \int_{\mathbf{R}}\left(s e^{x-\frac{1}{2} \Sigma}-K\right)^{+} \vartheta(x) \frac{1}{\sqrt{2 \pi \Sigma}} e^{-\frac{1}{2 \Sigma} x^{2}} d x+O\left(\epsilon^{2}\right),
\end{aligned}
$$

where $C^{B S}(T, K)$ represents a Black-Scholes call price and

$$
\vartheta(x)=E\left[\pi \mid \int_{0}^{T} \sigma_{t}^{(0)} d W_{1, t}=x\right]=\zeta\left(\frac{1}{\Sigma^{3}} x^{3}-\frac{1}{\Sigma^{2}} x^{2}-\frac{1}{\Sigma^{2}} 3 x+\frac{1}{\Sigma}\right) .
$$

Here, $\zeta$ and $\Sigma$ are some constants. (See Corollary A.1. in Appendix A for the detail.) Further, the integral in the last line of (68) can be explicitly given.

Consequently, our method is a natural extension of Fournié et al. (1999) and Siopacha and Teichmann (2010) in a sense that we obtain analytical approximations that do not rely on Monte Carlo simulations by making use of push-down of Malliavin weights. Although Lewis (2000) took a different approach, our method can be regarded as its extension because his models are included in our framework: in fact, Theorem 3.1 and 3.2 show that our method and formulas can be applied to multi-dimensional models including time-inhomogeneous ones in a unified way. That is, we can readily deal with multi-dimensional stochastic volatility models by the same procedure as in one-dimensional ones. A concrete example will be shown in 3.4.2 Double Heston model. In this regard, it seems not an easy task for other analytical approximations such as Lewis (2000), Hagan et al.(2002), Labordere (2008) and Antonelli-Scarlatti (2009) to apply the methods to higher dimensional models. 


\section{Numerical Examples}

\subsection{One-dimensional Stochastic Volatility Models}

This subsection shows numerical examples for the following stochastic volatility model:

$$
\begin{aligned}
& d S_{t}^{(\epsilon)}=\left(\sigma_{t}^{(\epsilon)}\right)^{\delta} S_{t}^{(\epsilon)} d W_{1, t}, \\
& d \sigma_{t}^{(\epsilon)}=\kappa\left(\theta-\sigma_{t}^{(\epsilon)}\right) d t+\epsilon\left(\sigma_{t}^{(\epsilon)}\right)^{\gamma}\left(\rho d W_{1, t}+\sqrt{1-\rho^{2}} d W_{2, t}\right),
\end{aligned}
$$

where all the parameters will be specified later. We set the risk-free interest rate to be zero ( i.e. $r=0$ ).

\section{Remark}

In Section 4, we deal with various stochastic volatility models including the Heston-type square-root volatility. (i.e. (69) with $\gamma=1 / 2$.) Although the square-root volatility model does not satisfy the regularity conditions stated in Section 3 since the coefficient function of the Heston-type model $A(\sigma)=\sqrt{\sigma}$ has an unbounded derivative at $\sigma=0$, our method is formally applicable.

A rigorous treatment for the application of our method to this case could be made in the similar manner as in Takahashi-Yoshida (2005) that utilizes a smooth modification technique: In fact, we take a modified process $\left(\tilde{\sigma}_{t}\right)_{t \in[0, T]}$ of square-root process $\left(\sigma_{t}\right)_{t \in[0, T]}$ :

$$
\begin{aligned}
& d S_{t}^{(\epsilon)}=\left(\sigma_{t}^{(\epsilon)}\right)^{\delta} S_{t}^{(\epsilon)} d W_{1, t}, \\
& d \sigma_{t}^{(\epsilon)}=\kappa\left(\theta-\sigma_{t}^{(\epsilon)}\right) d t+\epsilon \sqrt{\sigma_{t}^{(\epsilon)}}\left(\rho d W_{1, t}+\sqrt{1-\rho^{2}} d W_{2, t}\right), \\
& d \tilde{S}_{t}^{(\epsilon)}=\left(\sigma_{t}^{(\epsilon)}\right)^{\delta} \tilde{S}_{t}^{(\epsilon)} d W_{1, t}, \\
& d \tilde{\sigma}_{t}^{(\epsilon)}=\kappa\left(\theta-\tilde{\sigma}_{t}^{(\epsilon)}\right) d t+\epsilon g\left(\tilde{\sigma}_{t}^{(\epsilon)}\right)\left(\rho d W_{1, t}+\sqrt{1-\rho^{2}} d W_{2, t}\right) .
\end{aligned}
$$

Here, $g(x)$ is a smooth modification of $\sqrt{x}$ such that $g(x)=\sqrt{x}$ when $x \geq \epsilon_{1}$ for some small $\epsilon_{1}>0$, and $g(x)=0$ when $x \leq \epsilon_{2}$ for some $\epsilon_{2} \in\left(0, \epsilon_{1}\right)$. Specifically, we can set $g(x)$ as follows:

$$
\begin{aligned}
& g(x)=h(x) \sqrt{x} \\
& h(x)=\frac{\psi\left(x-\epsilon_{2}\right)}{\psi\left(x-\epsilon_{2}\right)+\psi\left(\epsilon_{1}-x\right)}, 0<\epsilon_{2}<\epsilon_{1} \\
& \psi(x)=e^{-1 / x} \text { for } x>0, \psi(x)=0 \text { for } x \leq 0 .
\end{aligned}
$$

Let $X_{T}^{(\epsilon)}=\log \left(S_{T}^{(\epsilon)} / s\right)$ and $\tilde{X}_{T}^{(\epsilon)}=\log \left(\tilde{S}_{T}^{(\epsilon)} / s\right)$. Then,

$$
\begin{aligned}
& X_{T}^{(\epsilon)}=\frac{-1}{2} \int_{0}^{T}\left(\sigma_{t}^{(\epsilon)}\right)^{2 \delta} d t+\int_{0}^{T}\left(\sigma_{t}^{(\epsilon)}\right)^{\delta} d W_{1, t} \\
& \tilde{X}_{T}^{(\epsilon)}=\frac{-1}{2} \int_{0}^{T}\left(\tilde{\sigma}_{t}^{(\epsilon)}\right)^{2 \delta} d t+\int_{0}^{T}\left(\tilde{\sigma}_{t}^{(\epsilon)}\right)^{\delta} d W_{1, t}
\end{aligned}
$$

Suppose that for a $\mathbf{R}$-valued function $f$ which for example stands for an option payoff; $f(x)=\left(e^{x}-K\right)^{+},\left(K-e^{x}\right)^{+}$, $E\left[\left|f\left(X_{T}^{(\epsilon)}\right)\right|^{2}\right]<\infty$ and $E\left[\left|f\left(\tilde{X}_{T}^{(\epsilon)}\right)\right|^{2}\right]<\infty$. Then, we have

$$
E\left[\left|f\left(X_{T}^{(\epsilon)}\right)-f\left(\tilde{X}_{T}^{(\epsilon)}\right)\right| 1_{\left\{\sigma^{(\epsilon)} \neq \tilde{\sigma}^{(\epsilon)}\right\}}\right] \leq\left(E\left[\left|f\left(X_{T}^{(\epsilon)}\right)\right|^{2}\right]^{\frac{1}{2}}+E\left[\left|f\left(\tilde{X}_{T}^{(\epsilon)}\right)\right|^{2}\right]^{\frac{1}{2}}\right) P\left(\left\{\sigma^{(\epsilon)} \neq \tilde{\sigma}^{(\epsilon)}\right\}\right)^{\frac{1}{2}} .
$$

It also holds that

$$
\begin{aligned}
& P\left(\left\{\sigma^{(\epsilon)} \neq \tilde{\sigma}^{(\epsilon)}\right\}\right)=P\left(\left\{\sigma_{t}^{(\epsilon)} \leq \epsilon_{1} \text { for some } t \in[0, T]\right\}\right) \\
& \leq P\left(\left\{\sup _{0 \leq t \leq T}\left|\sigma_{t}^{(\epsilon)}-\sigma_{t}^{(0)}\right|>a\right\}\right) \\
& +P\left(\left\{\sigma_{t}^{(\epsilon)} \leq \epsilon_{1} \text { for some } t \in[0, T]\right\} \cap\left\{\sup _{0 \leq t \leq T}\left|\sigma_{t}^{(\epsilon)}-\sigma_{t}^{(0)}\right| \leq a\right\}\right) .
\end{aligned}
$$

We can easily see that the second term after the last inequality is 0 . The first term is smaller than any $\epsilon^{n}$ for $n=1,2, \cdots$ by the following lemma of a large deviation inequality:

\section{Lemma}

Suppose that $Z_{t}^{(\epsilon)}, t \in[0, T]$ follows a SDE:

$$
d Z_{t}^{(\epsilon)}=a\left(Z_{t}^{(\epsilon)}\right) d t+\epsilon b\left(Z_{t}^{(\epsilon)}\right) d W_{t}
$$


where $a(z)$ satisfies Lipschitz and linear growth conditions, and $b(z)$ satisfies the linear growth condition. We assume that the unique strong solution exists. Then, there exists positive constants $c_{1}$ and $c_{2}$ independent of $\epsilon$ such that

for all $c>0$.

$$
P\left(\left\{\sup _{0 \leq s \leq T}\left|Z_{s}^{(\epsilon)}-Z_{s}^{(0)}\right|>c\right\}\right) \leq c_{1} \exp \left(-c_{2} \epsilon^{-2}\right)
$$

The lemma can be proved by slight modification of lemma 7.1 in Kunitomo and Takahashi (2003). Note also that $\sigma^{(\epsilon)}$ and $\tilde{\sigma}^{(\epsilon)}$ satisfy the conditions in the lemma above.

Hence,

$$
E\left[\left|f\left(X_{T}^{(\epsilon)}\right)-f\left(\tilde{X}_{T}^{(\epsilon)}\right)\right|\right]=o\left(\epsilon^{n}\right), n=1,2, \cdots .
$$

Therefore, the difference between $f\left(X_{T}^{(\epsilon)}\right)$ and $f\left(\tilde{X}_{T}^{(\epsilon)}\right)$ is negligible in the small disturbance asymptotic theory. Then, the mathematical conditions in Section 3 are met and our expansion method is rigorously applicable.

Hereafter, we use the following notations;

$$
\begin{aligned}
& n(x):=\frac{1}{\sqrt{2 \pi}} e^{-\frac{x^{2}}{2}}, \\
& N(x):=\int_{-\infty}^{x} n(y) d y \\
& \zeta:=\rho \int_{0}^{T} \delta\left(\sigma_{t}^{(0)}\right)^{(\delta-1)} e^{-\kappa t}\left(\sigma_{t}^{(0)}\right)^{\delta} \int_{0}^{t} e^{\kappa s}\left(\sigma_{s}^{(0)}\right)^{\gamma+\delta} d s d t \\
& \Sigma:=\int_{0}^{T}\left(\left(\sigma_{t}^{(0)}\right)^{\delta}\right)^{2} d t \\
& \vartheta(x):=E\left[\left.\frac{\partial}{\partial \epsilon} S_{T}^{(\epsilon)}\right|_{\epsilon=0} \int_{0}^{T} \frac{D_{t, 1} S_{T}^{(0)}}{\int_{0}^{T}\left(D_{s, 1} S_{T}^{(0)}\right)^{2} d s} d W_{1, t}-\left.\int_{0}^{T} D_{s, 1} \frac{\partial}{\partial \epsilon} S_{T}^{(\epsilon)}\right|_{\epsilon=0} \frac{D_{t, 1} S_{T}^{(0)}}{\int_{0}^{T}\left(D_{s, 1} S_{T}^{(0)}\right)^{2} d s} d t \mid \int_{0}^{T}\left(\sigma_{t}^{(0)}\right)^{\delta} d W_{1, t}=x\right] \\
& =\zeta\left(\frac{1}{\Sigma^{3}} x^{3}-\frac{1}{\Sigma^{2}} x^{2}-\frac{1}{\Sigma^{2}} 3 x+\frac{1}{\Sigma}\right) \text {, } \\
& \bar{\sigma}:=\left(\frac{1}{T} \int_{0}^{T}\left(\left(\sigma_{t}^{(0)}\right)^{\delta}\right)^{2} d t\right)^{1 / 2} \\
& d_{1}:=\frac{\log \left(\frac{s}{K}\right)}{\bar{\sigma} \sqrt{T}}+\frac{1}{2} \bar{\sigma} \sqrt{T} \\
& d_{2}:=\frac{\log \left(\frac{s}{K}\right)}{\bar{\sigma} \sqrt{T}}-\frac{1}{2} \bar{\sigma} \sqrt{T}, \\
& C^{B S}:=s N\left(d_{1}\right)-K N\left(d_{2}\right), \\
& C_{1}:=\int_{\mathbf{R}}\left(s e^{x-\frac{1}{2} \bar{\sigma}^{2} T}-K\right)^{+} \vartheta(x) \frac{1}{\sqrt{2 \pi \bar{\sigma}^{2} T}} \exp \left(-\frac{1}{2 \bar{\sigma}^{2} T} x^{2}\right) d x, \\
& \text { vega }:=s \sqrt{T} n\left(d_{1}\right) \text {. }
\end{aligned}
$$

\begin{tabular}{|c|c|c|c|}
\hline Strike price & Benchmark & Malliavin AE & Relative error \\
\hline$\overline{770}$ & 31.5478 & 31.5496 & $0.00 \%$ \\
\hline 80 & 23.6382 & 23.6471 & $0.04 \%$ \\
\hline 90 & 17.0487 & 17.0631 & $0.08 \%$ \\
\hline 100 & 11.8647 & 11.8816 & $0.14 \%$ \\
\hline 110 & 7.9947 & 8.0105 & $0.20 \%$ \\
\hline 120 & 5.2356 & 5.2474 & $0.23 \%$ \\
\hline
\end{tabular}

By using Corollary A.1 in Appendix A, the first order approximations of the asymptotic expansions of the option price and the implied volatility are given by

$$
\text { Malliavin AE }:=C^{B S}+\epsilon C_{1},
$$

and

$$
\text { Malliavin IV }:=\bar{\sigma}+\epsilon \frac{C_{1}}{v e g a}
$$

First, we give the numerical results on the accuracy of our approximation formula for call option prices. 
Benchmark : Heston's Fourier transform solutions.

$$
T=5, \delta=1, \gamma=1, s=100, \sigma_{0}=0.4, \kappa=0.1, \theta=0.4, \epsilon=0.2, \rho=-0.5
$$

\begin{tabular}{|c|c|c|c|}
\hline Strike price & Benchmark & Malliavin AE & Relative error \\
\hline \hline 70 & 46.4585 & 46.3591 & $-0.21 \%$ \\
80 & 41.5476 & 41.4427 & $-0.25 \%$ \\
90 & 37.1770 & 37.0591 & $-0.31 \%$ \\
100 & 33.2892 & 33.1521 & $-0.41 \%$ \\
110 & 29.8338 & 29.6697 & $-0.55 \%$ \\
120 & 26.7639 & 26.5645 & $-0.74 \%$ \\
\hline
\end{tabular}

Benchmark : Monte Carlo simulation (1,000,000 trials, 500 time steps).

$$
T=10, \delta=1, \gamma=1, s=100, \sigma_{0}=0.4, \kappa=0.1, \theta=0.4, \epsilon=0.2, \rho=-0.5
$$

\begin{tabular}{|c|c|c|c|}
\hline Strike price & Benchmark & Malliavin AE & Relative error \\
\hline \hline 70 & 55.3842 & 55.2118 & $-0.31 \%$ \\
80 & 51.4141 & 51.2058 & $-0.10 \%$ \\
90 & 47.8183 & 47.5671 & $-0.53 \%$ \\
100 & 44.5521 & 44.2513 & $-0.68 \%$ \\
110 & 41.5785 & 41.2207 & $-0.86 \%$ \\
120 & 38.8648 & 38.4432 & $-1.10 \%$ \\
\hline
\end{tabular}

Benchmark : Monte Carlo simulation (3,000,000 trials, 1000 time steps).

Next, we show the numerical results for implied volatilities.

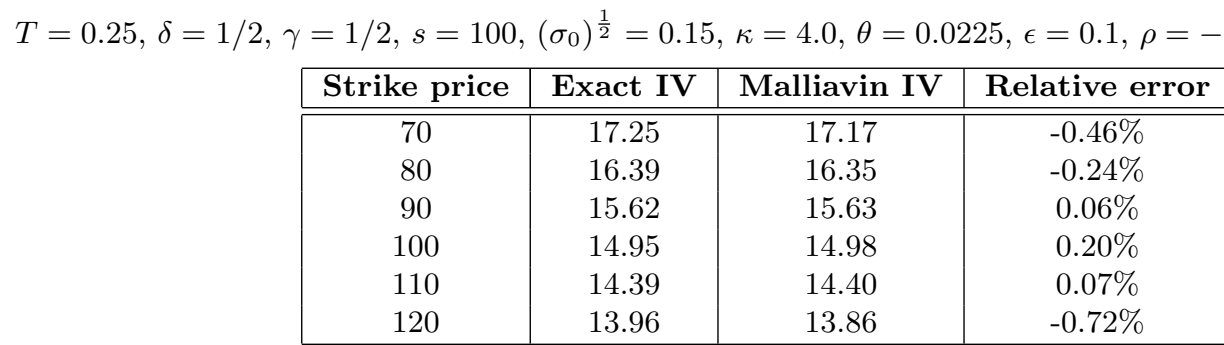

$T=0.5, \delta=1 / 2, \gamma=1 / 2, s=100,\left(\sigma_{0}\right)^{\frac{1}{2}}=0.4, \kappa=2.5, \theta=0.16, \epsilon=0.1, \rho=-0.5$

\begin{tabular}{|c|c|c|c|}
\hline Strike price & Exact IV & Malliavin IV & Relative error \\
\hline \hline 70 & 40.63 & 40.34 & $-0.71 \%$ \\
80 & 40.30 & 40.20 & $-0.26 \%$ \\
90 & 40.02 & 40.07 & $0.13 \%$ \\
100 & 39.78 & 39.96 & $0.45 \%$ \\
110 & 39.58 & 39.85 & $0.69 \%$ \\
120 & 39.41 & 39.76 & $0.89 \%$ \\
\hline
\end{tabular}

$T=1, \delta=1 / 2, \gamma=1 / 2, s=100,\left(\sigma_{0}\right)^{1 / 2}=0.2, \kappa=2.0, \theta=0.04, \epsilon=0.1, \rho=-0.25$

\begin{tabular}{|c|c|c|c|}
\hline Strike price & Exact IV & Malliavin IV & Relative error \\
\hline \hline 70 & 20.68 & 20.60 & $-0.40 \%$ \\
80 & 20.36 & 20.36 & $0.00 \%$ \\
90 & 20.10 & 20.15 & $0.26 \%$ \\
100 & 19.90 & 19.96 & $0.32 \%$ \\
110 & 19.75 & 19.80 & $0.23 \%$ \\
120 & 19.63 & 19.64 & $0.06 \%$ \\
\hline
\end{tabular}

\subsection{Double Heston Model}

Our method is general enough to be applied to multi-dimensional models. For instance, Gatheral (2008) introduced the following double stochastic volatility model;

$$
\begin{aligned}
d S_{t}^{(\epsilon)} & =r S_{t}^{(\epsilon)} d t+\sqrt{v_{t}^{(\epsilon)}} S_{t}^{(\epsilon)} d W_{1, t} \\
d v_{t}^{(\epsilon)} & =\kappa\left(\bar{v}_{t}^{(\epsilon)}-v_{t}^{(\epsilon)}\right) d t+\epsilon \sqrt{v_{t}^{(\epsilon)}}\left(\rho_{1} d W_{1, t}+\sqrt{1-\rho_{1}^{2}} d W_{2, t}\right) \\
d \bar{v}_{t}^{(\epsilon)} & =\bar{\kappa}\left(\theta-\bar{v}_{t}^{(\epsilon)}\right) d t+\epsilon \sigma_{2} \sqrt{\bar{v}_{t}^{(\epsilon)}}\left(\rho_{2} d W_{1, t}+\sqrt{1-\rho_{2}^{2}} d W_{3, t}\right)
\end{aligned}
$$


with $\rho_{1}, \rho_{2} \in[-1,1]$.

We derive an approximation formula for the double Heston model and show some numerical examples. Let $C^{\text {Double.SV }}$ and $\sigma^{I V}(T, K)$ be the call option and the implied volatility under the double Heston model;

$$
\begin{aligned}
C^{\text {Double.SV }}(T, K) & =e^{-r T} E\left[\left(S_{T}^{(\epsilon)}-K\right)^{+}\right], \\
C^{B S}\left(T, K, \sigma^{I V}(T, K)\right) & =C^{\text {Double.SV }}(T, K) .
\end{aligned}
$$

We have the following approximation formulas for $C^{\text {Double.SV }}$ and $\sigma^{I V}(T, K)$.

\section{Proposition 4.1}

$$
\begin{aligned}
C^{\text {Double.SV }}(T, K) & =C^{B S}(T, K, \bar{\sigma})+\epsilon_{1} C_{11}+\epsilon_{2} C_{12}+O\left(\epsilon^{2}\right) \\
\sigma^{I V}(T, K) & =\bar{\sigma}+\epsilon_{1} \frac{1}{\text { vega }} C_{11}+\epsilon_{2} \frac{1}{\text { vega }} C_{12}+O\left(\epsilon^{2}\right)
\end{aligned}
$$

where $\epsilon_{1}=\epsilon, \epsilon_{2}=\epsilon \sigma_{2}$,

$$
\begin{aligned}
C_{1 i} & =e^{-r T} \int_{\mathbf{R}}\left(s e^{x+r T-\frac{1}{2} \Sigma}-K\right)^{+} \eta_{i}\left(\frac{1}{\Sigma^{3}} x^{3}-\frac{1}{\Sigma^{2}} x^{2}-\frac{1}{\Sigma^{2}} 3 x+\frac{1}{\Sigma}\right) \frac{1}{\sqrt{2 \pi \Sigma}} e^{-\frac{1}{2 \Sigma} x^{2}} d x(i=1,2), \\
\eta_{1} & =\frac{1}{2} \rho_{1} \int_{0}^{t} e^{-\kappa s} \int_{0}^{s} e^{\kappa u} v_{u}^{(0)} d u d s \\
\eta_{2} & =\frac{1}{2} \rho_{2} \int_{0}^{t} \kappa e^{-\kappa s} \int_{0}^{s} e^{\bar{\kappa} u} \sqrt{\bar{v}_{u}^{(0)}} \int_{u}^{s} e^{(\kappa-\bar{\kappa}) v} d v \sqrt{v_{u}^{(0)}} d u d s, \\
\bar{\sigma} & =\left(\theta+\left(v_{0}^{(0)}-\theta\right) \frac{\left(1-e^{-\kappa T}\right)}{\kappa T}+\left(\bar{v}_{0}-\theta\right) \frac{\kappa}{\kappa-\bar{\kappa}}\left(\frac{\left(1-e^{-\bar{\kappa} T}\right)}{\bar{\kappa} T}-\frac{\left(1-e^{-\kappa T}\right)}{\kappa T}\right)\right)^{1 / 2}, \\
\text { vega } & =s \sqrt{T} n\left(d_{1}\right),
\end{aligned}
$$

with $\kappa \neq \bar{\kappa}$.

(Proof)

By the asymptotic expansion with push-down of the Malliavin weights, we have

$$
\begin{aligned}
C^{\text {Double.SV }}(T, K) & =e^{-r T} E\left[\left(S_{T}^{(\epsilon)}-K\right)^{+}\right] \\
& =e^{-r T} E\left[\left(S_{T}^{(0)}-K\right)^{+}\right]+\epsilon e^{-r T} E\left[\left(S_{T}^{(0)}-K\right)^{+} H\left(S_{T}^{(0)},\left.\frac{\partial}{\partial \epsilon} S_{t}^{(\epsilon)}\right|_{\epsilon=0}\right)\right]+O\left(\epsilon^{2}\right) \\
& =C^{B S}(T, K, \bar{\sigma})+\epsilon e^{-r T} \int_{\mathbf{R}}\left(s e^{x+r T-\frac{1}{2} \Sigma}-K\right)^{+} \vartheta(x) \frac{1}{\sqrt{2 \pi \Sigma}} e^{-\frac{x^{2}}{2 \Sigma}} d x+O\left(\epsilon^{2}\right),
\end{aligned}
$$

where

$$
\vartheta(x)=E\left[H\left(S_{T}^{(0)},\left.\frac{\partial}{\partial \epsilon} S_{t}^{(\epsilon)}\right|_{\epsilon=0}\right) \mid \int_{0}^{T} \sqrt{v_{s}^{(0)}} d W_{1, s}=x\right] .
$$

$\left.\frac{\partial}{\partial \epsilon} S_{t}^{(\epsilon)}\right|_{\epsilon=0}$ is given by

$$
\left.\frac{\partial}{\partial \epsilon} S_{t}^{(\epsilon)}\right|_{\epsilon=0}=S_{t}^{(0)} X_{t}
$$

where

$$
X_{t}=\left(\left.\int_{0}^{t} \frac{1}{2 \sqrt{v_{s}^{(0)}}} \frac{\partial}{\partial \epsilon} v_{s}^{(\epsilon)}\right|_{\epsilon=0} d W_{1, s}-\left.\frac{1}{2} \int_{0}^{t} \frac{\partial}{\partial \epsilon} v_{s}^{(\epsilon)}\right|_{\epsilon=0} d s\right)
$$

Then, $\vartheta$, the push-down of Malliavin weight is computed as follows;

$$
\begin{aligned}
\vartheta(x) & =E\left[\left(D^{*}(Z)-D_{Z}\right) \circ \frac{\partial}{\partial \epsilon} S_{t}^{(\epsilon)}||_{\epsilon=0} \mid \int_{0}^{T} \sqrt{v_{s}^{(0)}} d W_{1, s}=x\right] \\
& =\left(\frac{x}{\Sigma}-\frac{\partial}{\partial x}\right) \circ E\left[X_{T} \mid \int_{0}^{T} \sqrt{v_{s}^{(0)}} d W_{1, s}=x\right] \\
& =\left(\zeta_{1}+\zeta_{2}\right)\left(\frac{1}{\Sigma^{3}} x^{3}-\frac{1}{\Sigma^{2}} x^{2}-\frac{1}{\Sigma^{2}} 3 x+\frac{1}{\Sigma}\right),
\end{aligned}
$$


where

$$
\begin{aligned}
Z & =\frac{D_{1} S_{T}^{(0)}}{\left\|D_{1} S_{T}^{(0)}\right\|_{H}^{2}}, \\
\zeta_{1} & =\frac{1}{2} \rho_{1} \int_{0}^{t} e^{-\kappa s} \int_{0}^{s} e^{\kappa u} v_{u}^{(0)} d u d s \\
\zeta_{2} & =\frac{1}{2} \rho_{2} \sigma_{2} \int_{0}^{t} \kappa e^{-\kappa s} \int_{0}^{s} e^{\bar{\kappa} u} \sqrt{\bar{v}_{u}^{(0)}} \int_{u}^{s} e^{(\kappa-\bar{\kappa}) v} d v \sqrt{v_{u}^{(0)}} d u d s .
\end{aligned}
$$

Therefore, we have

$$
\begin{aligned}
& C^{\text {Double.SV }}(T, K) \\
= & C^{B S}(T, K, \bar{\sigma})+\epsilon e^{-r T} \int_{\mathbf{R}}\left(s e^{x+r T-\frac{1}{2} \Sigma}-K\right)^{+}\left(\zeta_{1}+\zeta_{2}\right)\left(\frac{1}{\Sigma^{3}} x^{3}-\frac{1}{\Sigma^{2}} x^{2}-\frac{1}{\Sigma^{2}} 3 x+\frac{1}{\Sigma}\right) \frac{1}{\sqrt{2 \pi \Sigma}} e^{-\frac{1}{2 \Sigma} x^{2}} d x \\
& +O\left(\epsilon^{2}\right) .
\end{aligned}
$$

By the similar argument as in 3.2, the implied volatility is expanded as follows:

$$
\begin{aligned}
& \sigma^{I V}(T, K) \\
= & \bar{\sigma}+\epsilon \frac{1}{v e g a} e^{-r T} \int_{\mathbf{R}}\left(s e^{x+r T-\frac{1}{2} \Sigma}-K\right)^{+}\left(\zeta_{1}+\zeta_{2}\right)\left(\frac{1}{\Sigma^{3}} x^{3}-\frac{1}{\Sigma^{2}} x^{2}-\frac{1}{\Sigma^{2}} 3 x+\frac{1}{\Sigma}\right) \frac{1}{\sqrt{2 \pi \Sigma}} e^{-\frac{1}{2 \Sigma} x^{2}} d x \\
+ & O\left(\epsilon^{2}\right) .
\end{aligned}
$$

Next, we show the numerical results on the accuracy of our approximation formula for the call option prices under the double Heston model. Also, in the tables below we define Malliavin AE and Malliavin IV as follows:

$$
\begin{aligned}
& \text { Malliavin AE }:=C^{B S}+\epsilon_{1} C_{11}+\epsilon_{2} C_{12}, \\
& \text { Malliavin IV }:=\bar{\sigma}+\epsilon_{1} \frac{C_{11}}{\text { vega }}+\epsilon_{2} \frac{C_{12}}{\text { vega }} .
\end{aligned}
$$

$$
T=1, s=100, \sigma_{0}=v_{0}^{1 / 2}=0.4, \kappa=0.1, \epsilon_{1}=0.1, \rho_{1}=-0.5, \bar{\kappa}=1.0, \theta=0.16, \epsilon_{2}=0.1, \rho_{2}=-0.25
$$

\begin{tabular}{|c|c|c|c|}
\hline Strike price & Benchmark & Malliavin AE & Relative error \\
\hline \hline 70 & 33.6198 & 33.5304 & $0.27 \%$ \\
80 & 26.5663 & 26.4797 & $0.33 \%$ \\
90 & 20.6084 & 20.5439 & $0.31 \%$ \\
100 & 15.7241 & 15.6937 & $0.19 \%$ \\
110 & 11.8225 & 11.8301 & $-0.06 \%$ \\
120 & 8.7741 & 8.8198 & $-0.52 \%$ \\
\hline
\end{tabular}

Benchmark : Monte Carlo simulation (1,000,000 trials, 500 time steps).

$$
T=2.5, s=100, \sigma_{0}=v_{0}^{1 / 2}=0.4, \kappa=0.1, \epsilon_{1}=0.1, \rho_{1}=-0.5, \bar{\kappa}=1.0, \theta=0.16, \epsilon_{2}=0.1, \rho_{2}=-0.25
$$

\begin{tabular}{|c|c|c|c|}
\hline Strike price & Benchmark & Malliavin AE & Relative error \\
\hline \hline 70 & 39.3315 & 39.4345 & $0.26 \%$ \\
80 & 33.5871 & 33.6975 & $0.33 \%$ \\
90 & 28.6053 & 28.7170 & $0.39 \%$ \\
100 & 24.3161 & 24.4228 & $0.44 \%$ \\
110 & 20.6430 & 20.7397 & $0.47 \%$ \\
120 & 17.5111 & 17.5926 & $0.47 \%$ \\
\hline
\end{tabular}

Benchmark : Monte Carlo simulation (1,000,000 trials, 500 time steps).

\subsection{Parameter Identification}

It is useful to see if the model parameter can be identified by our implied volatility expansion. For this purpose, we first compute the implied volatility for a stochastic volatility model with some fixed parameters by a numerical method such as Monte Carlo simulation. Next, we estimate the model parameters using our implied volatility expansion. As an example, we use the Heston model;

$$
\begin{aligned}
d X_{t} & =-\frac{v_{t}}{2} d t+\sqrt{v_{t}} d W_{1, t}, \\
d v_{t} & =\kappa\left(\theta-v_{t}\right) d t+\epsilon \sqrt{v_{t}}\left(\rho d W_{1, t}+\sqrt{1-\rho^{2}} d W_{2, t}\right),
\end{aligned}
$$


where $\rho \in[-1,1]$. Here, $\kappa$ and $\theta$ are known to be the speed and the level of the mean-reversion parameters, respectively. Also, the parameter $\nu=\epsilon \rho$ is regarded as a skewness parameter.

We compute the implied volatility with $S_{0}=e^{X_{0}}=100, v_{0}=0.04, \kappa=1.5, \theta=0.09$ and $\nu=-0.05$. Then, we obtain data set $\left\{\sigma^{M C}\left(T_{i}, K_{i j}\right)\right\}_{i, j}$ of the implied volatilities ( Exact IV ). Using our implied volatility expansion and the data set $\left\{\sigma^{M C}\left(T_{i}, K_{i j}\right)\right\}_{i, j}$, we solve the following minimization problem to estimate model parameters as if they were unknown:

$$
\min _{v_{0}, \kappa, \theta, \nu} \sum_{i=1}^{n} \sum_{j=1}^{m} \mid \sigma^{M C}\left(T_{i}, K_{i j}\right)-\text { Malliavin }\left.\mathbf{I V}\left(T_{i}, K_{i j}, v_{0}, \kappa, \theta, \nu\right)\right|^{2},
$$

where

$$
\text { Malliavin } \operatorname{IV}\left(T, K, v_{0}, \kappa, \theta, \nu\right)=\bar{\sigma}+\nu \frac{C_{1}}{v e g a}
$$

with

$$
\begin{aligned}
\bar{\sigma} & =\left(\theta+\left(v_{0}^{(0)}-\theta\right) \frac{\left(1-e^{-\kappa T}\right)}{\kappa T}\right)^{1 / 2} \\
C_{1} & =e^{-r T} \int_{\mathbf{R}}\left(s e^{x-\frac{1}{2} \Sigma}-K\right)^{+} \eta_{1}\left(\frac{1}{\Sigma^{3}} x^{3}-\frac{1}{\Sigma^{2}} x^{2}-\frac{1}{\Sigma^{2}} 3 x+\frac{1}{\Sigma}\right) \frac{1}{\sqrt{2 \pi \Sigma}} e^{-\frac{1}{2 \Sigma} x^{2}} d x, \\
\eta_{1} & =\frac{1}{2} \int_{0}^{t} e^{-\kappa s} \int_{0}^{s} e^{\kappa u} v_{u}^{(0)} d u d s .
\end{aligned}
$$

As a result, we obtain the optimal parameters $v_{0}^{*}=0.0404, \kappa^{*}=1.5022, \theta^{*}=0.0863$ and $\nu^{*}=-0.0336$, which are close to the true ones given above. Hence, the model parameters seems well identified by our implied volatility expansion for this case.

The fitting results are shown in the following tables, where Malliavin IV are computed by these optimal parameters $\left(v_{0}^{*}, \kappa^{*}, \theta^{*}, \nu^{*}\right)=(0.0404,1.5022,0.0863,-0.0336)$.

Monte Carlo parameter versus optimal parameter

\begin{tabular}{|c|c|c|}
\hline Parameters & Exact IV & Malliavin IV \\
\hline \hline$v_{0}$ & 0.0400 & 0.0404 \\
$\kappa$ & 1.5000 & 1.5022 \\
$\theta$ & 0.0900 & 0.0863 \\
$\nu$ & -0.0500 & -0.0336 \\
\hline
\end{tabular}

$T=0.4$ :

\begin{tabular}{|c|c|c|c|}
\hline Strike price & Exact IV & Malliavin IV & Relative error \\
\hline \hline 70 & 24.31 & 24.23 & $-0.32 \%$ \\
80 & 23.68 & 23.66 & $-0.05 \%$ \\
90 & 23.17 & 23.17 & $-0.01 \%$ \\
100 & 22.67 & 22.72 & $0.23 \%$ \\
110 & 22.30 & 22.32 & $0.09 \%$ \\
120 & 21.92 & 21.96 & $0.17 \%$ \\
\hline
\end{tabular}

$T=0.5$ :

\begin{tabular}{|c|c|c|c|}
\hline Strike price & Exact IV & Malliavin IV & Relative error \\
\hline \hline 70 & 24.71 & 24.74 & $0.13 \%$ \\
80 & 24.13 & 24.17 & $0.14 \%$ \\
90 & 23.64 & 23.66 & $0.06 \%$ \\
100 & 23.18 & 23.20 & $0.10 \%$ \\
110 & 22.83 & 22.79 & $-0.20 \%$ \\
120 & 22.48 & 22.41 & $-0.33 \%$ \\
\hline
\end{tabular}

\subsection{Calibration to Market Data}

In this subsection, we show calibration examples using an implied volatility data of Nikkei-225 option as of January 31,2011 , where the strikes are the closest to ATM with maturities $0.20,0.28,0.37,0.62,0.87,1.37$ and 1.87 years. Moreover, the data for ten different strikes other than ATM with maturity 0.87 year are also used since this kind of data is most available for the maturity. We compare the fitting result of our expansion in the single Heston model with that in the double Heston model. The single Heston model is expressed as

$$
\begin{aligned}
d X_{t} & =-\frac{v_{t}}{2} d t+\sqrt{v_{t}} d W_{1, t} \\
d v_{t} & =\kappa\left(\theta-v_{t}\right) d t+\epsilon_{1} \sqrt{v_{t}}\left(\rho_{1} d W_{1, t}+\sqrt{1-\rho_{1}^{2}} d W_{2, t}\right) .
\end{aligned}
$$


The double Heston model is expressed as

$$
\begin{aligned}
d X_{t} & =-\frac{v_{t}}{2} d t+\sqrt{v_{t}} d W_{1, t} \\
d v_{t} & =\kappa_{1}\left(\bar{v}_{t}-v_{t}\right) d t+\epsilon_{1} \sqrt{v_{t}}\left(\rho_{1} d W_{1, t}+\sqrt{1-\rho_{1}^{2}} d W_{2, t}\right) \\
d \bar{v}_{t} & =\kappa_{2}\left(\theta-\bar{v}_{t}\right) d t+\epsilon_{2} \sqrt{\bar{v}_{t}}\left(\rho_{2} d W_{1, t}+\sqrt{1-\rho_{2}^{2}} d W_{3, t}\right)
\end{aligned}
$$

with $W=\left(W_{1}, W_{2}, W_{3}\right)$ is a 3-dimensional Brownian motion. We define the skewness parameters $\nu_{1}$ and $\nu_{2}$ as $\nu_{1}=\epsilon_{1} \rho_{1}$ and $\nu_{2}=\epsilon_{2} \rho_{2}$. We also define Malliavin IV 1 and Malliavin IV 2 as

$$
\text { Malliavin IV } 1\left(T, K, v_{0}, \kappa_{1}, \theta, \nu_{1}\right):=\bar{\sigma}_{1}+\nu_{1} \frac{C_{11}}{v e g a}
$$

and

$$
\text { Malliavin IV } 2\left(T, K, v_{0}, \bar{v}_{0}, \kappa_{1}, \kappa_{2}, \theta, \nu_{1}, \nu_{2}\right):=\bar{\sigma}_{2}+\nu_{1} \frac{C_{11}}{\text { vega }}+\nu_{2} \frac{C_{12}}{\text { vega }},
$$

where $C_{1 i}(i=1,2)$ are previously given, and

$$
\begin{aligned}
& \bar{\sigma}_{1}=\left(\theta+\left(v_{0}^{(0)}-\theta\right) \frac{\left(1-e^{-\kappa T}\right)}{\kappa T}\right)^{1 / 2}, \\
& \bar{\sigma}_{2}=\left(\theta+\left(v_{0}^{(0)}-\theta\right) \frac{\left(1-e^{-\kappa T}\right)}{\kappa T}+\left(\bar{v}_{0}-\theta\right) \frac{\kappa}{\kappa-\bar{\kappa}}\left(\frac{\left(1-e^{-\bar{\kappa} T}\right)}{\bar{\kappa} T}-\frac{\left(1-e^{-\kappa T}\right)}{\kappa T}\right)\right)^{1 / 2} .
\end{aligned}
$$

To reduce the degree of freedom in Malliavin IV 2, we give the condition $v_{0}=\bar{v}_{0}=\theta$. For the Nikkei-225 data $\left\{\sigma^{\text {Data }}\left(T_{i}, K_{i j}\right)\right\}_{i, j}$, we solve the following minimization problems using our implied volatility expansions.

Optimization for Malliavin IV 1 (Single Heston model)

$$
\text { Error } \mathbf{1}=\min _{v_{0}, \kappa_{1}, \theta, \nu_{1}} \sum_{i=1}^{n} \sum_{j=1}^{m} \mid \sigma^{\text {Data }}\left(T_{i}, K_{i j}\right)-\text { Malliavin IV }\left.1\left(T_{i}, K_{i j}, v_{0}, \kappa_{1}, \theta, \nu_{1}\right)\right|^{2} .
$$

\begin{tabular}{|c|c|}
\hline Parameters & Malliavin IV 1 \\
\hline$v_{0}$ & 0.024 \\
\hline$\kappa_{1}$ & 2.805 \\
\hline$\theta$ & 0.0444 \\
\hline$\nu_{1}$ & -0.136 \\
\hline Parameters & Malliavin IV 2 \\
\hline$v_{0}$ & 0.038 \\
\hline$\kappa_{1}$ & 9.355 \\
\hline$\theta$ & 0.038 \\
\hline$\nu_{1}$ & -0.195 \\
\hline$\kappa_{2}$ & 9.070 \\
\hline$\nu_{2}$ & 0.580 \\
\hline
\end{tabular}

Optimization for Malliavin IV 2 (Double Heston model)

$$
\text { Error } \mathbf{2}=\min _{v_{0}, \kappa_{1}, \kappa_{2}, \theta, \nu_{1}, \nu_{2}} \sum_{i=1}^{n} \sum_{j=1}^{m} \mid \sigma^{D a t a}\left(T_{i}, K_{i j}\right)-\text { Malliavin IV }\left.2\left(T_{i}, K_{i j}, v_{0}, \kappa_{1}, \kappa_{2}, \theta, \nu_{1}, \nu_{2}\right)\right|^{2} .
$$

Then, we obtain the optimal parameters for the single and double Heston models, as shown in the following two tables.

The total differences between the calculated volatilities and the actual data are given as $\sqrt{\text { Error 1 }}=2.956 \%$ for Malliavin IV 1 and $\sqrt{\text { Error 2 }}=2.663 \%$ for Malliavin IV 2. Hence, the fitting result of the expansion of the double Heston is better than that of the single Heston. Also, the figures of the result for the double Heston model are shown in Figure 1 and 2.

\section{$5 \quad$ Applications}

This section provides approximation formulas for option prices under the shifted log-normal and jump-diffusion models with stochastic volatilities; an expansion of the implied volatility is also given for the jump-diffusion model. Also, for simplicity we set the risk-free interest rate $r=0$ in this section. 


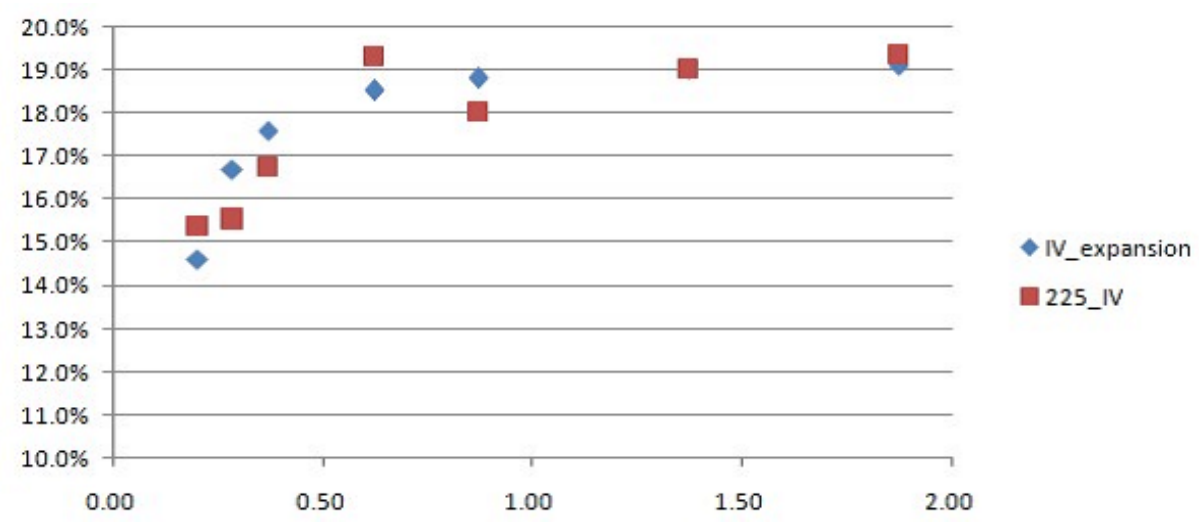

Figure 1: Term Structure

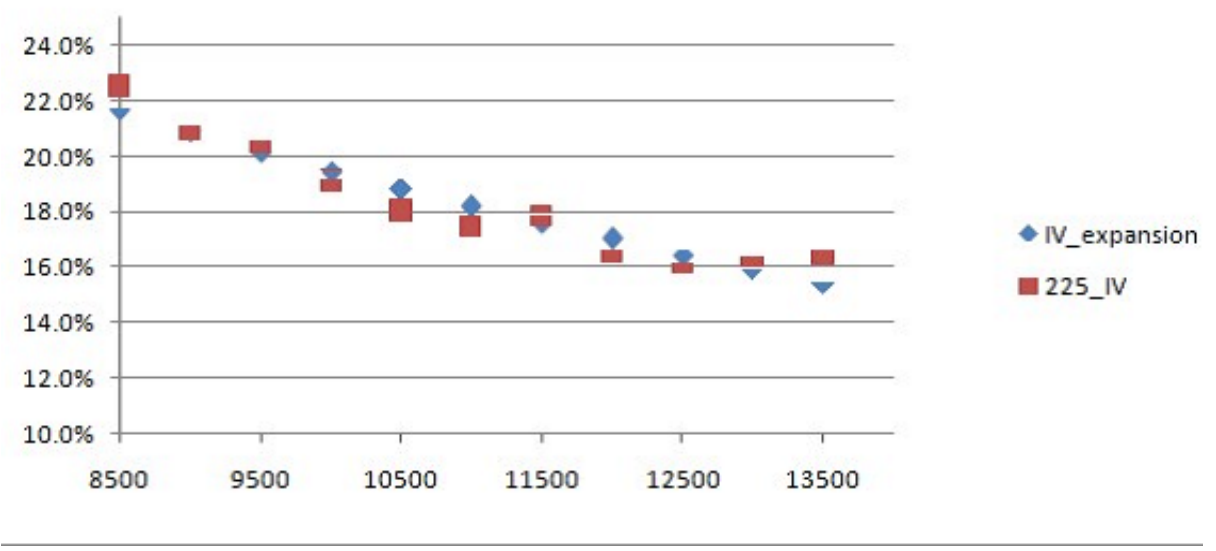

Figure 2: Skew

\subsection{Shifted Log-normal Model}

This subsection derives an approximation formula of the option price in the shifted log-normal model with stochastic volatility:

$$
\begin{aligned}
& d S_{t}^{(\epsilon)}=V\left(\sigma_{t}^{(\epsilon)}\right)\left(S_{t}^{(\epsilon)}-\beta\right) d W_{1, t} ; S_{0}^{(\epsilon)}=s>0, \\
& d \sigma_{t}^{(\epsilon)}=A_{0}\left(\sigma_{t}^{(\epsilon)}\right) d t+\epsilon A_{1}\left(\sigma_{t}^{(\epsilon)}\right)\left(\rho d W_{1, t}+\sqrt{1-\rho^{2}} d W_{2, t}\right),
\end{aligned}
$$

where $\beta$ is a constant such that $s>\beta$. At $\epsilon=0$, the option price is given by

$$
C^{B S}(\beta)=\int_{\mathbf{R}}\left((s-\beta) e^{x-\frac{1}{2} \Sigma}-(K-\beta)\right)^{+} \frac{1}{\sqrt{2 \pi \Sigma}} e^{-\frac{x^{2}}{2 \Sigma}} d x .
$$

We define the following deterministic process:

$$
\eta_{t}:=\exp \left\{\int_{0}^{t} A_{0}^{\prime}\left(\sigma_{u}^{(0)}\right) d u\right\}
$$

Then, the following proposition is obtained.

Proposition 5.1 An asymptotic expansion formula for the shifted log-normal model (92) is given as follows;

$$
C^{S V}(\beta)=C^{B S}(\beta)+\epsilon C_{1}(\beta)+O\left(\epsilon^{2}\right),
$$

where

$$
C_{1}(\beta)=\int_{\mathbf{R}}\left((s-\beta) e^{x-\frac{1}{2} \Sigma}-(K-\beta)\right)^{+} \vartheta(x) \frac{1}{\sqrt{2 \pi \Sigma}} e^{-\frac{x^{2}}{2 \Sigma}} d x
$$

with

$$
\begin{aligned}
\Sigma & =\int_{0}^{T} V\left(\sigma_{t}^{(0)}\right)^{2} d t \\
\vartheta(x) & =\zeta\left(\frac{1}{\Sigma^{3}} x^{3}-\frac{1}{\Sigma^{2}} x^{2}-\frac{1}{\Sigma^{2}} 3 x+\frac{1}{\Sigma}\right) \\
\zeta & =\rho \int_{0}^{T} \partial V\left(\sigma_{t}^{(0)}\right) \eta_{t} V\left(\sigma_{t}^{(0)}\right) \int_{0}^{t} \eta_{s}^{-1} A_{1}\left(\sigma_{s}^{(0)}\right) V\left(\sigma_{s}^{(0)}\right) d s d t .
\end{aligned}
$$


(Proof)

We first note that

$$
d S_{t}^{(\epsilon)}=d F_{t}^{(\epsilon)}=V\left(\sigma_{t}^{(\epsilon)}\right) F_{t}^{(\epsilon)} d W_{1, t} ; F_{0}^{(\epsilon)}=s-\beta>0,
$$

where $F_{t}^{(\epsilon)}=S_{t}^{(\epsilon)}-\beta$. Note also that $\left(S_{T}^{(\epsilon)}-K\right)^{+}=\left(F_{T}^{(\epsilon)}-(K-\beta)\right)^{+}$. Hence, in stead of the original problem we can consider the option pricing problem with the underlying asset price process $F^{(\epsilon)}$ and strike $K-\beta$. Then, the same argument as in Theorem 3.1 and Corollary A.1 can be applied to computation of the push-down of the Malliavin weight; $\vartheta(x):=E\left[\pi_{1} \mid \int_{0}^{T} V\left(\sigma_{t}^{(0)}\right) d W_{1, t}=x\right]$, where

$$
\begin{aligned}
\pi_{1} & =H_{1}\left(F_{T}^{(0)}, \Psi^{\beta_{1}}(T)\right) \\
\Psi^{\beta_{1}}(T) & =\left.\frac{\partial}{\partial \epsilon} F_{T}^{(\epsilon)}\right|_{\epsilon=0} .
\end{aligned}
$$

Thus, the result is obtained.

\subsection{Jump-Diffusion with Stochastic Volatility Model}

This subsection applies the Malliavin calculus to a jump-diffusion stochastic volatility (SVJ) model. Let $(\Omega, \mathcal{F}, P)$ be a probability space on which we define a Brownian motion $\left(W_{t}\right)_{t}$, a Poisson process $\left(N_{t}\right)_{t}$ with intensity $\lambda$ and i.i.d random variables $\left(\Delta_{j}\right)_{j \in \mathbf{N}}$ such that $\Delta_{j} \sim N(0,1)$. We will assume that the $\sigma$-algebras generated by $\left(W_{t}\right)_{t}$, $\left(N_{t}\right)_{t},\left(\Delta_{j}\right)_{j}$ are independent.

First, we introduce the perturbed stochastic differential equation : For $\epsilon \in[0,1]$,

$$
\begin{aligned}
& d S_{t}^{(\epsilon)}=V\left(\sigma_{t}^{(\epsilon)}\right) S_{t-}^{(\epsilon)} d W_{1, t}+S_{t-}^{(\epsilon)}\left(d J_{t}-\lambda m d t\right), \\
& S_{0}^{(\epsilon)}=s, \\
& d \sigma_{t}^{(\epsilon)}=A_{0}\left(\sigma_{t}^{(\epsilon)}\right) d t+\epsilon A_{1}\left(\sigma_{t}^{(\epsilon)}\right)\left(\rho d W_{1, t}+\sqrt{1-\rho^{2}} d W_{2, t}\right), \\
& \sigma_{0}^{(\epsilon)}=\sigma,
\end{aligned}
$$

where $J_{t}$ is defined by

$$
J_{t}=\sum_{j=1}^{N(t)}\left(e^{Y_{j}}-1\right),
$$

with $Y_{j}=a+b \Delta_{j}$ and $m=E\left[e^{Y_{j}}-1\right]$.

The solution of (99) is given by

$$
\begin{aligned}
S_{T} & =s \exp \left\{\int_{0}^{T} V\left(\sigma_{t}^{(\epsilon)}\right) d W_{1, t}-\frac{1}{2} \int_{0}^{T} V\left(\sigma_{t}^{(\epsilon)}\right)^{2} d t\right\} \prod_{j=1}^{N(T)} e^{Y_{j}} \\
& =s \exp \left\{\int_{0}^{T} V\left(\sigma_{t}^{(\epsilon)}\right) d W_{1, t}-\frac{1}{2} \int_{0}^{T} V\left(\sigma_{t}^{(\epsilon)}\right)^{2} d t+\sum_{j=1}^{N(T)} Y_{j}\right\} .
\end{aligned}
$$

We define the following notations:

$$
\begin{aligned}
X_{t}^{(\epsilon)} & :=\log \left(S_{t}^{(\epsilon)} / s\right) \\
\sigma^{B S} & :=\left(\frac{1}{T} \int_{0}^{T} V\left(\sigma_{t}^{(0)}\right)^{2} d t\right)^{1 / 2}, \\
\Sigma_{n} & :=\left(\int_{0}^{T} V\left(\sigma_{t}^{(0)}\right)^{2} d t+b^{2} n\right) \\
\sigma_{n} & :=\left(\frac{1}{T} \int_{0}^{T} V\left(\sigma_{t}^{(0)}\right)^{2} d t+\frac{b^{2} n}{T}\right)^{1 / 2}=\left(\left(\sigma^{B S}\right)^{2}+\left(b^{2} n / T\right)\right)^{1 / 2}, \\
\hat{r} & :=\frac{-\lambda m T+\left(a+b^{2} / 2\right) n}{T}, \\
d_{n} & :=\frac{1}{\sigma_{n} \sqrt{T}\left[\log \left(\frac{s}{K}\right)+\left(\hat{r}+\frac{\sigma_{n}^{2}}{2}\right) T\right],} \\
C_{n}^{B S}\left(T, K, \sigma_{n}\right) & :=s N\left(d_{n}\right)-e^{-\hat{r} T} K N\left(d_{n}-\sigma_{n} \sqrt{T}\right), \\
C^{M}\left(T, K, \sigma^{B S}\right) & :=\sum_{n=0}^{\infty} \frac{(\lambda(1+m) T)^{n} e^{-\lambda(1+m) T}}{n !} C_{n}^{B S}\left(T, K, \sigma_{n}\right), \\
C^{S V J}(T, K) & :=E\left[\left(S_{T}^{(\epsilon)}-K\right)^{+}\right] .
\end{aligned}
$$


Moreover, let us call $\sigma^{S V J . I V}(T, K)$ an implied volatility, which satisfies

$$
C^{S V J}(T, K)=C^{M}\left(T, K, \sigma^{S V J . I V}(T, K)\right) .
$$

Then, the following proposition is obtained.

Proposition 5.2 Under the jump-diffusion stochastic volatility (SVJ) model (99), the call option price $C^{S V J}(T, K)$ and its implied volatility $\sigma^{S V I . I V}(T, K)$ are expanded up to the $\epsilon$-order as follows:

$$
\begin{aligned}
C^{S V J}(T, K) & =C^{M}\left(T, K, \sigma^{B S}\right)+\epsilon C_{1}^{M}+O\left(\epsilon^{2}\right), \\
\sigma^{S V J . I V}(T, K) & =\sigma^{B S}+\epsilon \frac{1}{\operatorname{vega}^{M}} C_{1}^{M}+O\left(\epsilon^{2}\right),
\end{aligned}
$$

where

$$
\begin{aligned}
C_{1}^{M} & =\sum_{n=0}^{\infty} \frac{(\lambda T)^{n} e^{-\lambda T}}{n !} \int_{\mathbf{R}}\left(s e^{x-\lambda m T-\frac{1}{2} \int_{0}^{T} V\left(\sigma_{t}^{(0)}\right)^{2} d t+n a}-K\right)^{+} \vartheta_{n}(x) \frac{1}{\sqrt{2 \pi \Sigma_{n}}} e^{-\frac{1}{2 \Sigma_{n}} x^{2}} d x, \\
\operatorname{vega}^{M} & =\sum_{n=0}^{\infty} \frac{(\lambda(m+1) T)^{n} e^{-\lambda(m+1) T}}{n !} \frac{\sigma^{B S}}{\sigma_{n}} s \sqrt{T} n\left(d_{n}\right),
\end{aligned}
$$

with

$$
\begin{aligned}
\vartheta_{n}(x) & =\zeta\left(\frac{1}{\Sigma_{n}^{3}} x^{3}-\frac{1}{\Sigma_{n}^{2}} x^{2}-\frac{1}{\Sigma_{n}^{2}} 3 x+\frac{1}{\Sigma_{n}}\right) \\
\zeta & =\rho \int_{0}^{T} \partial V\left(\sigma_{t}^{(0)}\right) V\left(\sigma_{t}^{(0)}\right) \eta_{t} \int_{0}^{t} \eta_{s}^{-1} A_{1}\left(\sigma_{s}^{(0)}\right) V\left(\sigma_{s}^{(0)}\right) d s d t \\
\eta_{t} & =\exp \left\{\int_{0}^{t} A_{0}^{\prime}\left(\sigma_{u}^{(0)}\right) d u\right\} .
\end{aligned}
$$

(Proof)

See Appendix B.

\section{Conclusion}

This paper developed an asymptotic expansion method for generalized Wiener functionals based on the integrationby-parts formula in Malliavin calculus and the push-down of Malliavin weights. As an application, we derived asymptotic expansion formulas for option prices and implied volatilities as well as the density of the underlying asset price in stochastic volatility environment. we also presented numerical examples for expansions up to the first order ( $\epsilon$-order). Moreover, we applied the general formula to expansions of option prices for the shifted log-normal model with stochastic volatility. Further, combining some existing results of Malliavin calculus in jump-type models with our method, this paper we presented an approximation formula for the jump-diffusion model in stochastic volatility environment. More detailed numerical experiments and higher-order expansions are our next research topics.

\section{A Computation of Push-down of Malliavin Weights}

This section derives the push-down of Malliavin weights for the first and second order approximation terms of the asymptotic expansion of option prices.

We consider the following stochastic volatility model;

$$
\begin{aligned}
& d S_{t}^{(\epsilon)}=V\left(\sigma_{t}^{(\epsilon)}\right) S_{t}^{(\epsilon)} d W_{1, t}, \\
& d \sigma_{t}^{(\epsilon)}=A_{0}\left(\sigma_{t}^{(\epsilon)}\right) d t+\epsilon A_{1}\left(\sigma_{t}^{(\epsilon)}\right)\left(\rho d W_{1, t}+\sqrt{1-\rho^{2}} d W_{2, t}\right), \\
& S_{0}^{(\epsilon)}=S_{0}^{(0)}=s,
\end{aligned}
$$

where $V, A_{0}, A_{1} \in C_{b}^{\infty}(\mathbf{R}), \rho \in[-1,1]$ and $\epsilon \in[0,1]$.

Also we use the following notations;

$$
\begin{aligned}
B_{t} & =\rho W_{1, t}+\sqrt{1-\rho^{2}} W_{2, t}, \\
\eta_{t} & =\exp \left\{\int_{0}^{t} A^{\prime}\left(\sigma_{u}^{(0)}\right) d u\right\}, \\
\sigma_{t}^{(\epsilon)} & =\sigma_{t}^{(0)}+\epsilon \sigma_{t}^{(1)}+\epsilon^{2} \sigma_{t}^{(2)}+O\left(\epsilon^{3}\right), \\
\sigma_{0 t} & =\sigma_{t}^{(0)},
\end{aligned}
$$




$$
\begin{aligned}
\sigma_{t}^{(1)} & =\left.\frac{\partial}{\partial \epsilon} \sigma_{t}^{(\epsilon)}\right|_{\epsilon=0}=\int_{0}^{t} \eta_{t} \eta_{s}^{-1} A_{1}\left(\sigma_{0 s}\right) d B_{s} \\
\sigma_{t}^{(2)} & =\left.\frac{1}{2} \frac{\partial^{2}}{\partial \epsilon^{2}} \sigma_{t}^{(\epsilon)}\right|_{\epsilon=0}=\int_{0}^{t} \partial A_{1}\left(\sigma_{0 s}\right) \int_{0}^{s} \eta_{s} \eta_{u}^{-1} A_{1}\left(\sigma_{0 u}\right) d B_{u} d B_{s}, \\
v_{t}^{(0)} & =V\left(\sigma_{t}^{(0)}\right) \\
v_{t}^{(1)} & =V^{\prime}\left(\sigma_{0 t}\right) \sigma_{t}^{(1)}, \\
v_{t}^{(2)} & =2 V^{\prime}\left(\sigma_{0 t}\right) \sigma_{t}^{(2)}+V^{\prime \prime}\left(\sigma_{0 t}\right)\left(\sigma_{t}^{(1)}\right)^{2} . \\
X_{T}^{(0)} & =\int_{0}^{T} V\left(\sigma_{0 t}\right) d W_{1, t}-\frac{1}{2} \int_{0}^{T} V\left(\sigma_{0 t}\right)^{2} d t \\
X_{T}^{(1)} & =\left.\frac{\partial}{\partial \epsilon} X_{T}^{(\epsilon)}\right|_{\epsilon=0} \\
& =\int_{0}^{T} v_{t}^{(1)} d W_{1, t}-\int_{0}^{T} V\left(\sigma_{0 t}\right) v_{t}^{(1)} d t \\
X_{T}^{(2)} & =\left.\frac{\partial^{2}}{\partial \epsilon^{2}} X_{T}^{(\epsilon)}\right|_{\epsilon=0} \\
& =\int_{0}^{T} v_{t}^{(2)} d W_{1, t}-\int_{0}^{T}\left(v_{t}^{(1)}\right)^{2} d t-\int_{0}^{T} V\left(\sigma_{0 t}\right) v_{t}^{(2)} d t, \\
\bar{\sigma} & =\left(\frac{1}{T} \int_{0}^{T} V\left(\sigma_{t}^{(0)}\right)^{2} d t\right)^{1 / 2} .
\end{aligned}
$$

The closed-form approximation of the density and the call option price at $t=0$ with strike $K$ and maturity $T$ are given by

$$
\begin{aligned}
p^{S V}(y)= & p^{B S}(y)+\epsilon E\left[\pi_{1} \mid S_{T}^{(0)}=x\right] p^{B S}(y)+\epsilon^{2} E\left[\pi_{2} \mid S_{T}^{(0)}=x\right] p^{B S}(y)+O\left(\epsilon^{2}\right), \\
C^{S V}(T, K)= & C^{B S}(T, K, \bar{\sigma}) \\
& +\epsilon \int_{\mathbf{R}}(y-K)^{+} E\left[\pi_{1} \mid S_{T}^{(0)}=x\right] p^{B S}(y) d y \\
& +\epsilon^{2} \int_{\mathbf{R}}(y-K)^{+} E\left[\pi_{2} \mid S_{T}^{(0)}=x\right] p^{B S}(y) d y+O\left(\epsilon^{3}\right) \\
= & C^{B S}(T, K, \bar{\sigma}) \\
& +\epsilon \int_{\mathbf{R}}\left(s e^{x-\frac{1}{2} \int_{0}^{T} V\left(\sigma_{0 s}\right)^{2} d s}-K\right)^{+} E\left[\pi_{1} \mid \int_{0}^{T} V\left(\sigma_{0 t}\right) d W_{1, t}=x\right] n(\Sigma: x) d x \\
& +\epsilon^{2} \int_{\mathbf{R}}\left(s e^{x-\frac{1}{2} \int_{0}^{T} V\left(\sigma_{0 s}\right)^{2} d s}-K\right)^{+} E\left[\pi_{2} \mid \int_{0}^{T} V\left(\sigma_{0 t}\right) d W_{1, t}=x\right] n(\Sigma: x) d x+O\left(\epsilon^{3}\right) .
\end{aligned}
$$

where $\pi_{1}$ and $\pi_{2}$ is the Malliavin weights:

$$
\begin{aligned}
& \pi_{1}=H_{1}\left(S_{T}^{(0)},\left.\frac{\partial}{\partial \epsilon} S_{T}^{(\epsilon)}\right|_{\epsilon=0}\right), \\
& \pi_{2}=\frac{1}{2} H_{1}\left(S_{T}^{(0)},\left.\frac{\partial^{2}}{\partial \epsilon^{2}} S_{T}^{(\epsilon)}\right|_{\epsilon=0}\right)+\frac{1}{2} H_{2}\left(S_{T}^{(0)},\left(\left.\frac{\partial}{\partial \epsilon} S_{T}^{(\epsilon)}\right|_{\epsilon=0}\right)^{2}\right) .
\end{aligned}
$$

Note first that for $t \in[0, T], D_{t, 2} X_{T}^{(0)}=D_{t, 2} S_{T}^{(0)}=0, D_{t, 1} X_{T}^{(0)}=V\left(\sigma_{t}^{(0)}\right), D_{t, 1} S_{T}^{(0)}=S_{T}^{(0)} D_{t, 1} X_{T}^{(0)}=S_{T}^{(0)} V\left(\sigma_{t}^{(0)}\right)$ and $\left\|D S_{T}^{(0)}\right\|_{H}^{2}=\left\|D_{1} S_{T}^{(0)}\right\|_{H}^{2}=\left(S_{T}^{(0)}\right)^{2} \Sigma$ where $\Sigma=\int_{0}^{T} V\left(\sigma_{t}^{(0)}\right)^{2} d t$. Let $Z$ be the function on $L^{2}[0, T]$ defined by $Z(s)=\frac{D_{s, 1} X_{T}^{(0)}}{\left\|D_{1} X_{T}^{(0)}\right\|_{H}^{2}}=\frac{V\left(\sigma_{s}^{(0)}\right)}{\Sigma}$. Recall also that $D_{1}$ and $D_{1}^{*}$ are the Malliavin derivative and its adjoint operator (Skorohod integral) for the Brownian motion $W_{1}$. Then, the second-order Malliavin weight is computed as follows;

$$
\begin{aligned}
H_{1}\left(S_{T}^{(0)},\left.\frac{\partial^{2}}{\partial \epsilon^{2}} S_{T}^{(\epsilon)}\right|_{\epsilon=0}\right) & =D_{1}^{*}\left(\frac{D_{1} S_{T}^{(0)} S_{T}^{(0)}\left\{\left.\frac{\partial^{2}}{\partial \epsilon^{2}} X_{T}^{(\epsilon)}\right|_{\epsilon=0}+\left(\left.\frac{\partial}{\partial \epsilon} X_{T}^{(\epsilon)}\right|_{\epsilon=0}\right)^{2}\right\}}{\left\|D_{1} S_{T}^{(0)}\right\|_{H}^{2}}\right) \\
& =D_{1}^{*}\left(\frac{\left.D_{1} S_{T}^{(0)} S_{T}^{(0)} \frac{\partial^{2}}{\partial \epsilon^{2}} X_{T}^{(\epsilon)}\right|_{\epsilon=0}}{\left\|D_{1} S_{T}^{(0)}\right\|_{H}^{2}}\right)+D_{1}^{*}\left(\frac{D_{1} S_{T}^{(0)} S_{T}^{(0)}\left(\left.\frac{\partial}{\partial \epsilon} X_{T}^{(\epsilon)}\right|_{\epsilon=0}\right)^{2}}{\left\|D_{1} S_{T}^{(0)}\right\|_{H}^{2}}\right) \\
& =H_{1}\left(X_{T}^{(0)}, X_{T}^{(2)}\right)+H_{1}\left(X_{T}^{(0)},\left(X_{T}^{(1)}\right)^{2}\right) \\
& =X_{T}^{(2)} \int_{0}^{T} Z(s) d W_{1, s}-\int_{0}^{T} D_{s, 1} X_{T}^{(2)} Z(s) d s
\end{aligned}
$$




$$
\begin{aligned}
& +\left(X_{T}^{(1)}\right)^{2} \int_{0}^{T} Z(s) d W_{1, s}-\int_{0}^{T} D_{s, 1}\left(X_{T}^{(1)}\right)^{2} Z(s) d s, \\
& H_{2}\left(S_{T}^{(0)},\left(\left.\frac{\partial}{\partial \epsilon} S_{T}^{(\epsilon)}\right|_{\epsilon=0}\right)^{2}\right)=H_{1}\left(S_{T}^{(0)}, H_{1}\left(S_{T}^{(0)},\left(\left.\frac{\partial}{\partial \epsilon} S_{T}^{(\epsilon)}\right|_{\epsilon=0}\right)^{2}\right)\right) \\
= & D_{1}^{*}\left(\frac{D_{1} S_{T}^{(0)}}{\left\|D_{1} S_{T}^{(0)}\right\|_{H}^{2}} D_{1}^{*}\left(\frac{D_{1} S_{T}^{(0)}\left(\left.S_{T}^{(0)} \frac{\partial}{\partial \epsilon} X_{T}^{(\epsilon)}\right|_{\epsilon=0}\right)^{2}}{\left\|D_{1} S_{T}^{(0)}\right\|_{H}^{2}}\right)\right) \\
= & D_{1}^{*}\left(\frac{D_{1} S_{T}^{(0)}}{\left\|D_{1} S_{T}^{(0)}\right\|_{H}^{2}} D_{1}^{*}\left(\left(S_{T}^{(0)} X_{T}^{(1)}\right)^{2} Z\right)\right) \\
= & D_{1}^{*}\left(\frac{D_{1} S_{T}^{(0)}}{\left\|D_{1} S_{T}^{(0)}\right\|_{H}^{2}}\left\{S_{T}^{(0)}\left(X_{T}^{(1)}\right)^{2} \int_{0}^{T} Z(s) d W_{1, s}-\int_{0}^{T} D_{s, 1}\left(S_{T}^{(0)}\left(X_{T}^{(1)}\right)^{2}\right) Z(s) d s\right\}\right) \\
= & D_{1}^{*}\left(\frac{D_{1} S_{T}^{(0)}}{\left\|D_{1} S_{T}^{(0)}\right\|_{H}^{2}}\left\{S_{T}^{(0)}\left(X_{T}^{(1)}\right)^{2} \int_{0}^{T} Z(s) d W_{1, s}-\int_{0}^{T}\left(D_{1, s} S_{T}^{(0)}\right)\left(X_{T}^{(1)}\right)^{2} Z(s) d s-\int_{0}^{T} S_{T}^{(0)}\left(D_{s, 1}\left(X_{T}^{(1)}\right)^{2}\right) Z(s) d s\right\}\right) \\
= & D_{1}^{*}\left(\frac{D_{1} S_{T}^{(0)}}{\left\|D_{1} S_{T}^{(0)}\right\|_{H}^{2}}\left\{S_{T}^{(0)}\left(X_{T}^{(1)}\right)^{2} \int_{0}^{T} Z(s) d W_{1, s}-S_{T}^{(0)}\left(X_{T}^{(1)}\right)^{2} \int_{0}^{T} V\left(\sigma_{s}^{(0)}\right) Z(s) d s-S_{T}^{(0)} \int_{0}^{T}\left(D_{s, 1}\left(X_{T}^{(1)}\right)^{2}\right) Z(s) d s\right\}\right) \\
= & D_{1}^{*}\left(\frac{D_{1} X_{T}^{(0)}}{\left\|D_{1} X_{T}^{(0)}\right\|_{H}^{2}}\left\{\left(X_{T}^{(1)}\right)^{2} \int_{0}^{T} Z(s) d W_{1, s}-\int_{0}^{T}\left(D_{s, 1}\left(X_{T}^{(1)}\right)^{2}\right) Z(s) d s-\left(X_{T}^{(1)}\right)^{2}\right\}\right) \\
= & H_{2}\left(X_{T}^{(0)},\left(X_{T}^{(1)}\right)^{2}\right)-H_{1}\left(X_{T}^{(0)},\left(X_{T}^{(1)}\right)^{2}\right) .
\end{aligned}
$$

Then, we have

$$
\begin{aligned}
\pi_{2} & =\frac{1}{2} H_{1}\left(X_{T}^{(0)}, X_{T}^{(2)}\right)+\frac{1}{2} H_{2}\left(X_{T}^{(0)},\left(X_{T}^{(1)}\right)^{2}\right) \\
& =\frac{1}{2} H_{1}\left(X_{T}^{(0)}, \int_{0}^{T} v_{t}^{(2)} d W_{1, t}\right)-\frac{1}{2} H_{1}\left(X_{T}^{(0)}, \int_{0}^{T}\left(v_{t}^{(1)}\right)^{2} d t\right)-\frac{1}{2} H_{1}\left(X_{T}^{(0)}, \int_{0}^{T} V\left(\sigma_{0 t}\right) v_{t}^{(2)} d t\right) \\
& +\frac{1}{2} H_{2}\left(X_{T}^{(0)},\left(\int_{0}^{T} v_{t}^{(1)} d W_{1, t}\right)^{2}\right)-H_{2}\left(X_{T}^{(0)}, \int_{0}^{T} v_{t}^{(1)} d W_{1, t} \int_{0}^{T} V\left(\sigma_{0 t}\right) v_{t}^{(1)} d t\right)+\frac{1}{2} H_{2}\left(X_{T}^{(0)},\left(\int_{0}^{T} V\left(\sigma_{0 t}\right) v_{t}^{(1)} d t\right)^{2}\right) .
\end{aligned}
$$

In order to compute the expansion coefficient including the push-down of the Malliavin weights, we give the following formula (115) which is modified version of Malliavin (1997) and Malliavin-Thalmaier (2006).

Proposition A.1 For $\Psi \in \mathbf{D}_{\infty}(\mathcal{W}: \mathbf{R})$ and $Z=\frac{h}{\|h\|_{H}^{2}}$ with $h \in H$,

$$
E\left[D^{*}(Z) \Psi-D_{Z} \Psi \mid D^{*}(h)=x\right]=\frac{x}{\|h\|_{H}^{2}} E\left[\Psi \mid D^{*}(h)=x\right]-\frac{\partial}{\partial x} E\left[\Psi \mid D^{*}(h)=x\right] .
$$

(Proof)

We follow Proposition (8.2) in p.82 of Malliavin (1997).

Let $g$ be a smooth nondegenerate Wiener map, $g \in \mathbf{D}_{\infty}\left(\mathcal{W}: \mathbf{R}^{n}\right)$ and $z=\left(z^{1}, z^{2}, \cdots, z^{n}\right)$ be a vector field on $\mathbf{R}^{n}$. Suppose also that $Z=\left(Z^{1}, Z^{2}, \cdots, Z^{d}\right)$ be a lift of $z$ to the Wiener space ( a covering vector field of $z$ );

$$
Z^{k}(t)=\sum_{1 \leq s, l \leq n} \gamma^{s, l}\left(D_{t, k} g^{s}\right) z^{l}
$$

where $\gamma^{s, l}$ is the $(s, l)$-element of the inverse matrix of the Malliavin covariance matrix of $g$.

Then, Proposition (8.2) in p.82 of Malliavin (1997) says that for $\Psi \in \mathbf{D}_{\infty}(\mathcal{W}: \mathbf{R})$ and $k(x)=E^{g=x}[\Psi]$ where $E^{g=x}$ denotes the conditional expectation under $g=x$,

$$
E^{g=x}\left[D^{*}(Z) \Psi-D_{Z} \Psi\right]=k(x) E^{g=x}\left[D^{*}(Z)\right]-\partial_{z} k(x),
$$

where $\partial_{z} k(x):=\langle z,(d k(x) / d x)\rangle$. In our case, set $g=D^{*}(h)$ and then, $g \in \mathbf{D}_{\infty}(\mathcal{W}: \mathbf{R})$ (i.e. $\left.n=1\right)$. Also, let $z \equiv 1$ and hence,

$$
Z=\frac{D g}{\|D g\|_{H}^{2}}=\frac{h}{\|h\|_{H}^{2}} .
$$

Finally, note that $E^{g=x}\left[D^{*}(Z)\right]=\frac{x}{\|h\|_{H}^{2}}$ in our case, and thus the result (115) is obtained.

Using (115) above, we give the first order approximation formula of the call option price under the stochastic volatility. 
Corollary A.1 The first order approximation of the call price is given by

$$
C^{S V}(T, K)=C^{B S}(T, K, \bar{\sigma})+\epsilon \int_{\mathbf{R}}\left(s e^{x-\frac{1}{2} \Sigma}-K\right)^{+} \vartheta(x) \frac{1}{\sqrt{2 \pi \Sigma}} e^{-\frac{1}{2 \Sigma} x^{2}} d x,
$$

where

$$
\begin{aligned}
\vartheta(x) & =\zeta\left(\frac{1}{\Sigma^{3}} x^{3}-\frac{1}{\Sigma^{2}} x^{2}-\frac{1}{\Sigma^{2}} 3 x+\frac{1}{\Sigma}\right) \\
\Sigma & =\int_{0}^{T} V\left(\sigma_{s}^{(0)}\right)^{2} d s \\
\zeta & =\rho \int_{0}^{T} V^{\prime}\left(\sigma_{t}^{(0)}\right) \eta_{t} V\left(\sigma_{t}^{(0)}\right) \int_{0}^{t} \eta_{s}^{-1} A_{1}\left(\sigma_{s}^{(0)}\right) V\left(\sigma_{s}^{(0)}\right) d s d t
\end{aligned}
$$

with

$$
\eta_{t}=\exp \left\{\int_{0}^{t} A_{0}^{\prime}\left(\sigma_{u}^{(0)}\right) d u\right\}
$$

where $A_{0}^{\prime}\left(\sigma_{u}^{(0)}\right)=\left.\frac{d A_{0}(x)}{d x}\right|_{x=\sigma_{u}^{(0)}}$.

(Proof)

By (115) and the formula ${ }^{1}$;

$$
E\left[\int_{0}^{T} \int_{0}^{t} h_{2 u} d W_{1, u} h_{3 t} d W_{1, t} \mid \int_{0}^{T} h_{1 v} d W_{1, v}=x\right]=\left(\int_{0}^{T} \int_{0}^{t} h_{2 u} h_{1 u} d u h_{3 t} h_{1 t} d t\right)\left(\frac{x^{2}}{\Sigma^{2}}-\frac{1}{\Sigma}\right),
$$

for $h_{i} \in L^{2}[0, T], i=1,2,3$, The push-down of the Malliavin weight $H_{1}\left(X_{T}^{(0)}, \int_{0}^{T} v_{t}^{(1)} d W_{1, t}\right)$ is obtained as follows.

$$
\begin{aligned}
& E\left[H_{1}\left(X_{T}^{(0)}, \int_{0}^{T} v_{t}^{(1)} d W_{1, t}\right) \mid \int_{0}^{T} V\left(\sigma_{0 s}\right) d W_{1, s}=x\right] \\
= & E\left[\left(D^{*}(Z)-D_{Z}\right) \circ \int_{0}^{T} v_{t}^{(1)} d W_{1, t} \mid \int_{0}^{T} V\left(\sigma_{0 s}\right) d W_{1, s}=x\right] \\
= & \left(\frac{x}{\Sigma}-\frac{\partial}{\partial x}\right) \circ E\left[\int_{0}^{T} V^{\prime}\left(\sigma_{0 t}\right) \int_{0}^{t} \eta_{t} \eta_{s}^{-1} A_{1}\left(\sigma_{0 s}\right) d B_{s} d W_{1, t} \mid \int_{0}^{T} V\left(\sigma_{0 s}\right) d W_{1, s}=x\right] \\
= & \left(\frac{x^{3}}{\Sigma^{3}}-\frac{3 x}{\Sigma^{2}}\right) \zeta,
\end{aligned}
$$

where $\zeta=\rho \int_{0}^{T} V^{\prime}\left(\sigma_{0 t}\right) \eta_{t} V\left(\sigma_{0 t}\right) \int_{0}^{t} \eta_{s}^{-1} A_{1}\left(\sigma_{0 s}\right) V\left(\sigma_{0 s}\right) d s d t$

By (115) and the formula;

$$
E\left[\int_{0}^{T} h_{2 t} d W_{1, t} \mid \int_{0}^{T} h_{1 v} d W_{1, v}=x\right]=\left(\int_{0}^{T} h_{2 t} h_{1 t} d t\right) \frac{x}{\Sigma}
$$

the push-down of the Malliavin weight $H_{1}\left(X_{T}^{(0)}, \int_{0}^{T} V\left(\sigma_{0 t}\right) v_{t}^{(1)} d t\right)$ is obtained as

$$
\begin{aligned}
& E\left[H_{1}\left(X_{T}^{(0)}, \int_{0}^{T} V\left(\sigma_{0 t}\right) v_{t}^{(1)} d t\right) \mid \int_{0}^{T} V\left(\sigma_{0 s}\right) d W_{1, s}=x\right] \\
= & E\left[\left(D^{*}(Z)-D_{Z}\right) \circ \int_{0}^{T} V\left(\sigma_{0 t}\right) V^{\prime}\left(\sigma_{0 t}\right) \int_{0}^{t} \eta_{t} \eta_{s}^{-1} A_{1}\left(\sigma_{0 s}\right) d B_{s} d t \mid \int_{0}^{T} V\left(\sigma_{0 s}\right) d W_{1, s}=x\right] \\
= & E\left[\left(D^{*}(Z)-D_{Z}\right) \circ \int_{0}^{T} \eta_{s}^{-1} A_{1}\left(\sigma_{0 s}\right) \int_{t}^{T} V\left(\sigma_{0 t}\right) V^{\prime}\left(\sigma_{0 t}\right) \eta_{t} d t d B_{s} \mid \int_{0}^{T} V\left(\sigma_{0 s}\right) d W_{1, s}=x\right] \\
= & \left(\frac{x}{\Sigma}-\frac{\partial}{\partial x}\right) \circ E\left[\int_{0}^{T} \eta_{s}^{-1} A_{1}\left(\sigma_{0 s}\right) \int_{t}^{T} V\left(\sigma_{0 t}\right) V^{\prime}\left(\sigma_{0 t}\right) \eta_{t} d t d B_{s} \mid \int_{0}^{T} V\left(\sigma_{0 s}\right) d W_{1, s}=x\right] \\
= & \left(\frac{x^{2}}{\Sigma^{2}}-\frac{1}{\Sigma}\right) \zeta .
\end{aligned}
$$

Therefore, we obtain

$$
E\left[\pi_{1} \mid \int_{0}^{T} V\left(\sigma_{0 t}\right) d W_{1, t}=x\right]=\zeta\left(\frac{1}{\Sigma^{3}} x^{3}-\frac{1}{\Sigma^{2}} x^{2}-\frac{1}{\Sigma^{2}} 3 x+\frac{1}{\Sigma}\right) .
$$

\footnotetext{
${ }^{1}$ For the derivation and more general results, see Section 3 in Takahashi-Takehara-Toda (2009).
} 


\section{A.1 Second Order Approximation}

This subsection lists up the results necessary for computing push-down of the Malliavin weights of $\epsilon^{2}$-order terms of (114). The detail of the computation will be given upon request.

A.1.1 $H_{1}\left(X_{T}^{(0)}, \int_{0}^{T} v_{t}^{(2)} d W_{1, t}\right)$

$$
\begin{gathered}
E\left[H_{1}\left(X_{T}^{(0)}, \int_{0}^{T} v_{t}^{(2)} d W_{1, t}\right) \mid \int_{0}^{T} V\left(\sigma_{0 s}\right) d W_{1, s}=x\right] \\
=\left(\frac{x^{4}}{\Sigma^{4}}-\frac{6 x^{2}}{\Sigma^{3}}+\frac{3}{\Sigma^{2}}\right)\left(2 b_{11}+b_{12}\right)+\left(\frac{x^{2}}{\Sigma^{2}}-\frac{1}{\Sigma}\right) b_{13}
\end{gathered}
$$

where

$$
\begin{aligned}
b_{11} & =\rho^{2} \int_{0}^{T} V^{\prime}\left(\sigma_{0 t}\right) V\left(\sigma_{0 t}\right) \int_{0}^{t} A_{1}^{\prime}\left(\sigma_{0 s}\right) \eta_{s} V\left(\sigma_{0 s}\right) \int_{0}^{s} \eta_{u}^{-1} A_{1}\left(\sigma_{0 u}\right) V\left(\sigma_{0 u}\right) d u d s d t, \\
b_{12} & =\int_{0}^{T}\left(\int_{0}^{t} V\left(\sigma_{0 s}\right) \eta_{s}^{-1} A_{1}\left(\sigma_{0 s}\right) d s\right)^{2} V^{\prime \prime}\left(\sigma_{0 t}\right) \eta_{t}^{2} V\left(\sigma_{0 t}\right) d t, \\
b_{13} & =\int_{0}^{T} V^{\prime \prime}\left(\sigma_{0 t}\right) V\left(\sigma_{0 t}\right)\left(\int_{0}^{t} \eta_{t} \eta_{t}^{-1} A_{1}\left(\sigma_{0 s}\right)\right)^{2} d s d t .
\end{aligned}
$$

A.1.2 $H_{1}\left(X_{T}^{(0)}, \int_{0}^{T}\left(v_{t}^{(1)}\right)^{2} d t\right)$

$$
E\left[H_{1}\left(X_{T}^{(0)}, \int_{0}^{T}\left(v_{t}^{(1)}\right)^{2} d t\right) \mid \int_{0}^{T} V\left(\sigma_{0 s}\right) d W_{1, s}=x\right]=\left(\frac{x^{3}}{\Sigma^{3}}-\frac{3 x}{\Sigma^{2}}\right) b_{21}+\frac{x}{\Sigma} b_{22}
$$

where

$$
\begin{aligned}
b_{21} & =\rho^{2} \int_{0}^{T}\left(V^{\prime}\left(\sigma_{0 t}\right) \eta_{t}\right)^{2}\left(\int_{0}^{t} \eta_{s}^{-1} A_{1}\left(\sigma_{0 s}\right) V\left(\sigma_{0 s}\right) d s\right)^{2} d t \\
b_{22} & =\int_{0}^{T}\left(V^{\prime}\left(\sigma_{0 t}\right) \eta_{t}\right)^{2} \int_{0}^{t}\left(\eta_{s}^{-1} A_{1}\left(\sigma_{0 s}\right)\right)^{2} d s d t .
\end{aligned}
$$

A.1.3 $H_{1}\left(X_{T}^{(0)}, \int_{0}^{T} V\left(\sigma_{0 t}\right) v_{t}^{(2)} d t\right)$

$$
E\left[H_{1}\left(X_{T}^{(0)}, \int_{0}^{T} V\left(\sigma_{0 t}\right) v_{t}^{(2)} d t\right) \mid \int_{0}^{T} V\left(\sigma_{0 s}\right) d W_{1, s}=x\right]=2\left(\frac{x^{3}}{\Sigma^{3}}-\frac{3 x}{\Sigma^{2}}\right) b_{31}+\left(\frac{x^{3}}{\Sigma^{3}}-\frac{3 x}{\Sigma^{2}}\right) b_{32}+\frac{x}{\Sigma} b_{33},
$$

where

$$
\begin{aligned}
b_{31} & =\rho^{2} \int_{0}^{T} V^{\prime}\left(\sigma_{0 t}\right) V\left(\sigma_{0 t}\right) \int_{0}^{t} A_{1}^{\prime}\left(\sigma_{0 s}\right) \eta_{s} V\left(\sigma_{0 s}\right) \int_{0}^{s} \eta_{u}^{-1} A_{1}\left(\sigma_{0 u}\right) V\left(\sigma_{0 u}\right) d u d s d t, \\
b_{32} & =\rho^{2} \int_{0}^{T}\left(V^{\prime \prime}\left(\sigma_{0 t}\right) \eta_{t}^{2} V\left(\sigma_{0 t}\right)\right)\left(\int_{0}^{t} \eta_{s}^{-1} A_{1}\left(\sigma_{0 s}\right) V\left(\sigma_{0 s}\right) d s\right)^{2} d t, \\
b_{33} & =\int_{0}^{T}\left(V^{\prime \prime}\left(\sigma_{0 t}\right) \eta_{t}^{2} V\left(\sigma_{0 t}\right)\right) \int_{0}^{t}\left(\eta_{s}^{-1} A_{1}\left(\sigma_{0 s}\right)\right)^{2} d s d t .
\end{aligned}
$$

A.1.4 $H_{2}\left(X_{T}^{(0)},\left(\int_{0}^{T} v_{t}^{(1)} d W_{1, t}\right)^{2}\right)$

$$
\begin{aligned}
& E\left[H_{2}\left(X_{T}^{(0)},\left(\int_{0}^{T} v_{t}^{(1)} d W_{1, t}\right)^{2}\right) \mid \int_{0}^{T} V\left(\sigma_{0 s}\right) d W_{1, s}=x\right] . \\
= & \left(\frac{x^{6}}{\Sigma^{6}}-\frac{15 x^{4}}{\Sigma^{5}}+\frac{45 x^{2}}{\Sigma^{4}}-\frac{15}{\Sigma^{3}}\right) b_{41}+\left(\frac{x^{4}}{\Sigma^{4}}-\frac{6 x^{2}}{\Sigma^{3}}+\frac{3}{\Sigma}\right)\left(2 b_{42}+2 b_{43}+b_{44}\right)+\left(\frac{x^{2}}{\Sigma^{2}}-\frac{1}{\Sigma^{2}}\right) b_{45},
\end{aligned}
$$


where

$$
\begin{aligned}
b_{41} & =\left(\rho \int_{0}^{T} V^{\prime}\left(\sigma_{0 t}\right) \eta_{t} V\left(\sigma_{0 t}\right) \int_{0}^{t} \eta_{s}^{-1} A_{1}\left(\sigma_{0 s}\right) V\left(\sigma_{0 s}\right) d s d t\right)^{2}, \\
b_{42} & =\int_{0}^{T} V^{\prime}\left(\sigma_{0 t}\right) \eta_{t} V\left(\sigma_{0 t}\right) \int_{0}^{t} V^{\prime}\left(\sigma_{0 s}\right) \eta_{s} V\left(\sigma_{0 s}\right) \int_{0}^{s}\left(\eta_{u}^{-1} A_{1}\left(\sigma_{0 u}\right)\right)^{2} d u d s d t, \\
b_{43} & =\rho^{2} \int_{0}^{T} V^{\prime}\left(\sigma_{0 t}\right) \eta_{t} V\left(\sigma_{0 t}\right) \int_{0}^{t} V^{\prime}\left(\sigma_{0 s}\right) A_{1}\left(\sigma_{0 s}\right) \int_{0}^{s} V\left(\sigma_{0 u}\right) \eta_{u}^{-1} A_{1}\left(\sigma_{0 u}\right) d u d s d t, \\
b_{44} & =\rho^{2} \int_{0}^{T}\left(V^{\prime}\left(\sigma_{0 t}\right) \eta_{t}\right)^{2}\left(\int_{0}^{t} \eta_{s}^{-1} A_{1}\left(\sigma_{0 s}\right) V\left(\sigma_{0 s}\right) d s\right)^{2} d t \\
b_{45} & =\int_{0}^{T}\left(V^{\prime}\left(\sigma_{0 t}\right) \eta_{t}\right)^{2} \int_{0}^{t}\left(\eta_{s}^{-1} A_{1}\left(\sigma_{0 s}\right)\right)^{2} d s d t .
\end{aligned}
$$

A.1.5 $H_{2}\left(X_{T}^{(0)}, \int_{0}^{T} v_{t}^{(1)} d W_{t} \int_{0}^{T} V\left(\sigma_{0 t}\right) v_{t}^{(1)} d t\right)$

$$
\begin{aligned}
& E\left[H_{2}\left(X_{T}^{(0)}, \int_{0}^{T} v_{t}^{(1)} d W_{1, t} \int_{0}^{T} V\left(\sigma_{0 t}\right) v_{t}^{(1)} d t\right) \mid \int_{0}^{T} V\left(\sigma_{0 t}\right) d W_{1, t}=x\right] \\
= & \left(\frac{x^{5}}{\Sigma^{5}}-\frac{10 x^{3}}{\Sigma^{4}}+\frac{15 x}{\Sigma^{3}}\right) b_{51}+\left(\frac{x^{3}}{\Sigma^{3}}-\frac{3 x}{\Sigma^{2}}\right)\left(b_{52}+b_{53}\right),
\end{aligned}
$$

where

$$
\begin{aligned}
b_{51} & =\rho^{2}\left(\int_{0}^{T} q_{3 t} q_{1 t} \int_{0}^{t} q_{2 s} q_{1 s} d s d t\right)\left(\int_{0}^{T} q_{4 u}\left(\int_{u}^{T} q_{5 s} d s\right) q_{1 u} d u\right) \\
b_{52} & =\left(\int_{0}^{T} q_{3 t} q_{1 t} \int_{0}^{t} q_{4 s} q_{2 s}\left(\int_{s}^{T} q_{5 u} d u\right) d s d t\right) \\
b_{53} & =\left(\rho^{2} \int_{0}^{T} q_{4 t} q_{3 t}\left(\int_{t}^{T} q_{5 u} d u\right) \int_{0}^{t} q_{2 s} q_{1 s} d s d t\right) \\
q_{1 t} & =V\left(\sigma_{0 t}\right) \\
q_{2 t} & =q_{4 t}=\eta_{t}^{-1} A_{1}\left(\sigma_{0 t}\right) \\
q_{3 t} & =V^{\prime}\left(\sigma_{0 t}\right) \eta_{t} \\
q_{5 t} & =V^{\prime}\left(\sigma_{0 t}\right) V\left(\sigma_{0 t}\right) \eta_{t} .
\end{aligned}
$$

A.1.6 $H_{2}\left(X_{T}^{(0)},\left(\int_{0}^{T} V\left(\sigma_{0 t}\right) v_{t}^{(1)} d t\right)^{2}\right)$

$$
E\left[H_{2}\left(X_{T}^{(0)},\left(\int_{0}^{T} V\left(\sigma_{0 t}\right) v_{t}^{(1)} d t\right)^{2}\right) \mid \int_{0}^{T} V\left(\sigma_{0 s}\right) d W_{1, s}=x\right]=2\left[\left(\frac{x^{4}}{\Sigma^{4}}-\frac{6 x^{2}}{\Sigma^{3}}+\frac{3}{\Sigma}\right) b_{61}+\left(\frac{x^{2}}{\Sigma^{2}}-\frac{1}{\Sigma}\right) b_{62}\right],
$$

where

$$
\begin{aligned}
b_{61} & =\rho^{2} \int_{0}^{T} q_{3 t}\left(\int_{0}^{t} q_{2 u} q_{1 u} d u\right)\left(\int_{0}^{t}\left(\int_{u}^{t} q_{3 s} d s\right) q_{2 u} q_{1 u} d u\right) d t \\
b_{62} & =\int_{0}^{T} q_{3 t} \int_{0}^{t} q_{2 u}^{2}\left(\int_{u}^{t} q_{3 s} d s\right) d u d t \\
q_{1 t} & =V\left(\sigma_{0 t}\right) \\
q_{2 t} & =\eta_{t}^{-1} A_{1}\left(\sigma_{0 t}\right), \\
q_{3 t} & =V^{\prime}\left(\sigma_{0 t}\right) V\left(\sigma_{0 t}\right) \eta_{t} .
\end{aligned}
$$

\section{B Proof of Proposition 5.2}

Let $C_{1}^{M}$ denote the coefficient of $\epsilon$-order in the asymptotic expansion of $C^{S V J}$ around $\epsilon=0$ :

$$
C_{1}^{M}=\left.\frac{\partial}{\partial \epsilon} E\left[\left(S_{T}^{(\epsilon)}-K\right)^{+}\right]\right|_{\epsilon=0}
$$




$$
\begin{aligned}
& =E\left[\left.\partial_{x}\left\{\left(S_{T}^{(0)}-K\right)^{+}\right\} \frac{\partial}{\partial \epsilon} S_{T}^{(\epsilon)}\right|_{\epsilon=0}\right] \\
& =\sum_{n=0}^{\infty} E\left[\left.\partial_{x}\left\{\left(S_{T}^{(0)}-K\right)^{+}\right\} \frac{\partial}{\partial \epsilon} S_{T}^{(\epsilon)}\right|_{\epsilon=0} \mathbf{1}_{\{N(T)=n\}}\right],
\end{aligned}
$$

where

$$
\partial_{x}\left\{\left(S_{T}^{(0)}-K\right)^{+}\right\}=\left.\frac{\partial}{\partial x}(x-K)^{+}\right|_{x=S_{T}^{(0)}} .
$$

Next, by the integration by parts formula including the jump amplitudes (e.g. Bavouzet and Messaoud (2006)), the right hand side of the last equality in the above equation is expressed as

$$
C_{1}^{M}=\sum_{n=0}^{\infty} E\left[\left(S_{T}^{(0)}-K\right)^{+} H_{1, n} \mathbf{1}_{\{N(T)=n\}}\right],
$$

where $H_{1, n}$ is the Malliavin weight on $\{N(T)=n\}$, which will be given below:

Let $D_{0}$ be the Malliavin derivative with respect to Brownian motion for $W_{1}$ and $D_{i}$ be the Malliavin derivative with respect to the jump amplitudes $\Delta_{i}, i=1, \cdots, n$. Then, on $\{N(T)=n\}$,

$$
\begin{gathered}
D_{0, t} S_{T}^{(0)}=S_{T}^{(0)} D_{0 t} X_{T}^{(0)}=S_{T}^{(0)} V\left(\sigma_{t}^{(0)}\right), t \in[0, T], \\
D_{i} S_{T}^{(0)}=S_{T}^{(0)} D_{i} X_{T}^{(0)}=S_{T}^{(0)} b, i=1, \cdots, n, \\
X_{T}^{(0)}=\int_{0}^{T} V\left(\sigma_{t}^{(0)}\right) d W_{1, t}-\frac{1}{2} \int_{0}^{T} V\left(\sigma_{t}^{(0)}\right)^{2} d t+\sum_{j=1}^{n} Y_{j},
\end{gathered}
$$

where we use $D_{i} X_{T}^{(0)}=b$ (e.g. see p.280 in Bavouzet-Messaoud (2006)) as well as $D_{0, t} X_{T}^{(0)}=V\left(\sigma_{t}^{(0)}\right)$.

Then, the Malliavin covariance of $S_{T}^{(0)}, \sigma_{S_{T}^{(0)}}$ is given by

$$
\begin{aligned}
\sigma_{S_{T}^{(0)}} & =\int_{0}^{T}\left(D_{0, t} S_{T}^{(0)}\right)^{2} d t+\sum_{i=1}^{n}\left(D_{i} S_{T}^{(0)}\right)^{2} \\
& =\left(S_{T}^{(0)}\right)^{2} \int_{0}^{T} V\left(\sigma_{t}^{(0)}\right)^{2} d t+\left(S_{T}^{(0)}\right)^{2} b^{2} n \\
& =\left(S_{T}^{(0)}\right)^{2} \Sigma_{n},
\end{aligned}
$$

where

$$
\Sigma_{n}:=\int_{0}^{T} V\left(\sigma_{t}^{(0)}\right)^{2} d t+\left(S_{T}^{(0)}\right)^{2} b^{2} n
$$

and

$$
\sum_{i=1}^{n}\left(D_{i} S_{T}^{(0)}\right)^{2}=\left(S_{T}^{(0)}\right)^{2}\left(b^{2} n\right)
$$

is the contribution by the jump part. (e.g. See p.282 in Bavouzet-Messaoud (2006).)

Using the Skorohod integral for the Brownian motion and the jump amplitude, on $\{N(T)=n\}$ we can compute the Malliavin weight $H_{1, n}$ as follows;

$$
\begin{aligned}
H_{1, n} & =\sum_{i=0}^{n} D_{i}^{*}\left(\frac{\left.\frac{\partial}{\partial \epsilon} S_{T}^{(\epsilon)}\right|_{\epsilon=0} D_{i} S_{T}^{(0)}}{\sigma_{S_{T}^{(0)}}}\right)=\sum_{i=0}^{n} D_{i}^{*}\left(\frac{S_{T}^{(0)} X_{T}^{(1)} S_{T}^{(0)} D_{i} X_{T}^{(0)}}{\left(S_{T}^{(0)}\right)^{2} \Sigma_{n}}\right) \\
& =\frac{1}{\Sigma_{n}} \sum_{i=0}^{n} D_{i}^{*}\left(X_{T}^{(1)} D_{i} X_{T}^{(0)}\right) \\
& =\frac{1}{\Sigma_{n}}\left(X_{T}^{(1)} D_{0}^{*}\left(D_{0} X_{T}^{(0)}\right)-\int_{0}^{T}\left(D_{0, t} X_{T}^{(1)}\right)\left(D_{0, t} X_{T}^{(0)}\right) d t\right) \\
& +\frac{1}{\Sigma_{n}} \sum_{i=1}^{n}\left(\left(X_{T}^{(1)} D_{i}^{*}\left(D_{i} X_{T}^{(0)}\right)-\left(D_{i} X_{T}^{(1)}\right)\left(D_{i} X_{T}^{(0)}\right)\right),\right.
\end{aligned}
$$

where each $D_{i}^{*}(i=0,1, \cdots, n)$ is the adjoint operator of $D_{i}(i=0,1, \cdots, n)$ and

$$
\begin{aligned}
X_{T}^{(1)} & =\left.\frac{\partial}{\partial \epsilon} X_{T}^{(\epsilon)}\right|_{\epsilon=0} \\
& =\int_{0}^{T} V^{\prime}\left(\sigma_{0 t}\right) \int_{0}^{t} \eta_{t} \eta_{s}^{-1} A_{1}\left(\sigma_{0 s}\right) d B_{s} d W_{1, t}-\int_{0}^{T} V\left(\sigma_{0 t}\right) V^{\prime}\left(\sigma_{0 t}\right) \int_{0}^{t} \eta_{t} \eta_{s}^{-1} A_{1}\left(\sigma_{0 s}\right) d B_{s} d t,
\end{aligned}
$$


with

$$
B_{t}=\rho W_{1, t}+\sqrt{1-\rho^{2}} W_{2, t}
$$

Note that $D_{0}^{*}\left(D_{0} X_{T}^{(0)}\right)$ is expressed by the usual Itô integral,

$$
D_{0}^{*}\left(D_{0} X_{T}^{(0)}\right)=\int_{0}^{T} V\left(\sigma_{t}^{(0)}\right) d W_{1, t}
$$

Recall that $\Delta_{i}$ is Gaussian random variable $N(0,1)$. Hence, from p.280 in Bavouzet-Messaoud $(2006), D_{i}^{*}\left(D_{i} X_{T}^{(0)}\right)$ for $i=1, \cdots, n$ is given by

$$
D_{i}^{*}\left(D_{i} X_{T}^{(0)}\right)=D_{i}^{*}(b)=-b \frac{\partial}{\partial \Delta_{i}} \log p\left(\Delta_{i}\right)=b \Delta_{i}
$$

where

$$
p(x)=\frac{1}{\sqrt{2 \pi}} e^{-\frac{x^{2}}{2}}
$$

Because $X_{T}^{(1)}$ is a stochastic integral driven by the Brownian motion which is independent of $\Delta_{i}$, we have for $t \in[0, T]$,

$$
\begin{aligned}
D_{0, t} X_{T}^{(1)}= & V^{\prime}\left(\sigma_{t}^{(0)}\right) \int_{0}^{t} \eta_{t} \eta_{s}^{-1} A_{1}\left(\sigma_{s}^{(0)}\right) d B_{s}+\rho \eta_{t}^{-1} A_{1}\left(\sigma_{t}^{(0)}\right) \int_{t}^{T} V^{\prime}\left(\sigma_{u}^{(0)}\right) \eta_{u} d W_{1, u} \\
& -\rho \eta_{t}^{-1} A_{1}\left(\sigma_{t}^{(0)}\right) \int_{t}^{T} V^{\prime}\left(\sigma_{u}^{(0)}\right) V\left(\sigma_{u}^{(0)}\right) \eta_{u} d u
\end{aligned}
$$

and for $i=1, \cdots, n$,

$$
D_{i} X_{T}^{(1)}=0
$$

Hence, (140) is expressed as

$$
H_{1, n}=\frac{1}{\Sigma_{n}}\left(X_{T}^{(1)}\left(\int_{0}^{T} V\left(\sigma_{t}^{(0)}\right) d W_{1, t}+\sum_{i=1}^{n} b \Delta_{i}\right)-\int_{0}^{T} D_{0, t} X_{T}^{(1)} V\left(\sigma_{t}^{(0)}\right) d t\right) .
$$

Then,

$$
\begin{aligned}
C_{1}^{M} & =\sum_{n=0}^{\infty} E\left[\left(S_{T}^{(0)}-K\right)^{+} H_{1, n} \mathbf{1}_{\{N(T)=n\}}\right] \\
& =\sum_{n=0}^{\infty} \frac{(\lambda T)^{n} e^{-\lambda T}}{n !} E\left[\left(S_{T}^{(0)}-K\right)^{+} H_{1, n}\right] \\
& =\sum_{n=0}^{\infty} \frac{(\lambda T)^{n} e^{-\lambda T}}{n !} \int_{\mathbf{R}}\left(s e^{x-\lambda m T-\frac{1}{2} \int_{0}^{T} V\left(\sigma_{t}^{(0)}\right)^{2} d t+n a}-K\right)^{+} \vartheta_{n}(x) \frac{1}{\sqrt{2 \pi \Sigma_{n}}} e^{-\frac{1}{2 \Sigma_{n}} x^{2}} d x
\end{aligned}
$$

where $\vartheta_{n}(x)$ is the push down of the Malliavin weight $H_{1, n}$;

$$
\vartheta_{n}(x)=E\left[H_{1, n} \mid \int_{0}^{T} V\left(\sigma_{t}^{(0)}\right) d W_{1, t}+\sum_{i=1}^{n} b \Delta_{i}=x\right] .
$$

We easily evaluate $\vartheta_{n}(x)$ as

$$
\vartheta_{n}(x)=\zeta\left(\frac{1}{\Sigma_{n}^{3}} x^{3}-\frac{1}{\Sigma_{n}^{2}} x^{2}-\frac{1}{\Sigma_{n}^{2}} 3 x+\frac{1}{\Sigma_{n}}\right) .
$$

Thus, we obtain (104):

$$
C^{S V J}(T, K)=C^{M}\left(T, K, \sigma^{B S}\right)+\epsilon C_{1}^{M}+O\left(\epsilon^{2}\right) .
$$

Next, let us regard $C^{M}$ as a function of $\sigma^{B S}$ :

$$
\sigma^{B S} \mapsto C^{M}\left(T, K, \sigma^{B S}\right)=\sum_{n=0}^{\infty} \frac{(\lambda(m+1) T)^{n} e^{-\lambda(m+1) T}}{n !} C_{n}^{B S}\left(\left(\left(\sigma^{B S}\right)^{2}+\left(b^{2} n / T\right)\right)^{1 / 2}\right) .
$$


Also, the vega of $C^{M}$ with respect to $\sigma^{B S}$, vega ${ }^{M}$ is given by

$$
\begin{aligned}
\text { vega }^{M} & =\frac{\partial}{\partial \sigma^{B S}} C^{M}\left(T, K, \sigma^{B S}\right) \\
& =\sum_{n=0}^{\infty} \frac{(\lambda(m+1) T)^{n} e^{-\lambda(m+1) T}}{n !} \frac{\sigma^{B S}}{\sigma_{n}} s \sqrt{T} n\left(d_{n}\right) .
\end{aligned}
$$

Suppose that $\sigma^{S V J . I V}$ satisfies

$$
C^{S V J}(T, K)=C^{M}\left(T, K, \sigma^{S V J . I V}(T, K)\right),
$$

and that $\sigma^{S V J . I V}(T, K)$ is expanded around $\epsilon=0$ :

$$
\sigma^{S V J . I V}(T, K)=\sigma^{B S}+\epsilon \cdot \sigma^{1}+O\left(\epsilon^{2}\right) .
$$

Then, we have

$$
C^{M}\left(\sigma^{S V J . I V}\right)=C^{M}\left(T, K, \sigma^{B S}\right)+\epsilon \cdot v e g a^{M} \sigma^{1}+O\left(\epsilon^{2}\right) .
$$

Finally, comparing (104) with (147), we obtain

$$
\sigma^{1}=\frac{C_{1}^{M}}{\operatorname{vega}^{M}}
$$

which leads to the result (105).

\section{References}

[1] Antonelli, F. and Scarlatti, S.: Pricing Options under Stochastic Volatility: a Power Series Approach, Finance Stoch. (2009), no. 13, 269-303.

[2] Bavouzet, M-P. and Messaoud, M.: Computation of Greeks Using Malliavin's Calculus in Jump Type Market Models, Electronic Journal of Probability. 11 (2006), no. 10, 276-300.

[3] Ben Arous, G. and Laurence, P.: Second Order Expansion for Implied Volatility in Two Factor Local Stochastic Volatility Models and Applications to the Dynamic $\lambda$-SABR Model, Preprint (2009)

[4] Fouque, J-P., Papanicolaou G. and Sircar, K.R.: Derivatives in Financial Markets with Stochastic Volatility, Cambridge University Press, Cambridge, (2000).

[5] Fournié, E., Lasry, J.-M., Lebuchoux, J., Lions, P.-L. and Touzi, N.: Applications of Malliavin Calculus to Monte-Carlo Methods in Finance, Finance Stoch. 3 (1999), no. 4, 391-412.

[6] Fournié, E., Lebuchoux, J. and Touzi, N.: Small Noise Expansion and Importance Sampling, Asymptotic Anal. (1997), 361-376.

[7] Gatheral, J.: Further Developments in Volatility Derivatives Modeling, Global Derivatives Trading \& Risk Management, Paris, (2008)

[8] Gatheral, J., Hsu, E.P. and Laurence, P., Ouyang, C. and Wang, T-H.: Asymptotics of Implied Volatility in Local Volatility Models: forthcoming in Mathematical Finance (2009)

[9] Hagan, P.S., Kumar, D., Lesniewskie, A.S. and Woodward, D.E.: Managing Smile Risk, Wilmott magazine, (2002).

[10] Ikeda, N. and Watanabe, S.: Stochastic Differential Equations and Diffusion Processes, Second Edition, NorthHolland/Kodansha. (1989)

[11] Kunitomo, N. and Takahashi, A.: On Validity of the Asymptotic Expansion Approach in Contingent Claim Analysis, Annals of Applied Probability, Vol.13, No.3, (2003).

[12] Labordére, P.H.: Analysis, Geometry and Modeling in Finance : Advanced Methods in Options Pricing, Chapman and Hall, (2008).

[13] Lewis, A.L.: Option Valuation under Stochastic Volatility with Mathematica Code, Newport Beach, CA: Finance Press. (2000)

[14] Malliavin, P. and Thalmaier, A.: Stochastic Calculus of Variations in Mathematical Finance, Springer. (2006)

[15] Malliavin, P.: Stochastic Analysis, Springer. (1997)

[16] Nualart, D.: The Malliavin Calculus and Related Topics, Springer. (2006)

[17] Sakamoto, Y. and Yoshida, N.: Asymptotic Expansions of Mixture Type Statistics Based on Generalized Wiener Functionals, Cooperative Research Report 58, (1994) The Insitute of Statistical Mathematics, Tokyo, Japan. 
[18] Shiraya, K., Takahashi, A. and Yamada, T.: Pricing Discrete Barrier Options under Stochastic Volatility, forthcoming in Asia-Pacific Financial Markets (2010)

[19] Siopacha, M. and Teichmann, J.: Weak and Strong Taylor Methods for Numerical Solutions of Stochastic Differential Equations, Quantitative Finance. 11(4), (2010) 517-528.

[20] Takahashi, A.: Essays on the Valuation Problems of Contingent Claims, Ph.D. Dissertation, Haas School of Business. (1995) University of California, Berkeley.

[21] Takahashi, A.: An Asymptotic Expansion Approach to Pricing Financial Contingent Claims, Asia-Pacific Financial Markets 6 (1999), 115-151

[22] Takahashi, A., Takehara, K. and Toda, M.: Computation in an Asymptotic Expansion Method, Preprint, CARF Working Paper Series CARF-F-149. (2009)

[23] Takahashi, A. and Yoshida, N.: An Asymptotic Expansion Scheme for Optimal Investment Problems, Statistical Inference for Stochastic Processes 7 (2004), 153-188.

[24] Takahashi, A. and Yoshida, N.: Monte Carlo Simulation with Asymptotic Method, The Journal of Japan Statistical Society, Vol. 35-2, (2005), 171-203.

[25] Watanabe, S.: Malliavin's Calculus in Terms of Generalized Wiener Functionals, Lecture Notes in Control and Inform. Sci., 49, Springer. (1983)

[26] Watanabe, S.: Lectures on Stochastic Differential Equations and Malliavin Calculus, Springer. (1984)

[27] Watanabe, S.: Analysis of Wiener Functionals (Malliavin Calculus) and its Applications to Heat Kernels, Ann. Probab. 15 (1987), no. 1, 1-39.

[28] Yoshida, N.: Asymptotic Expansion for Small Diffusions via the Theory of Malliavin-Watanabe, Probability Theory and Related Fields, 92, (1992a), 275-311.

[29] Yoshida, N.: Asymptotic Expansion for Statistics Related to Small Diffusions, J.Japan Statist. Soc. 22 (1992b), no.2, 139-159. 